\title{
Dynamical Bulk Scaling Limit of Gaussian Unitary Ensembles and Stochastic Differential Equation Gaps
}

\author{
Yosuke Kawamoto $^{1} \cdot$ Hirofumi Osada $^{1}$ (D)
}

Received: 24 May 2017 / Published online: 14 February 2018

(C) The Author(s) 2019, corrected publication 2019

\begin{abstract}
The distributions of $N$-particle systems of Gaussian unitary ensembles converge to $\mathrm{Sine}_{2}$ point processes under bulk scaling limits. These scalings are parameterized by a macro-position $\theta$ in the support of the semicircle distribution. The limits are always $\mathrm{Sine}_{2}$ point processes and independent of the macro-position $\theta$ up to the dilations of determinantal kernels. We prove a dynamical counterpart of this fact. We prove that the solution to the $N$-particle system given by a stochastic differential equation (SDE) converges to the solution of the infinite-dimensional Dyson model. We prove that the limit infinite-dimensional SDE (ISDE), referred to as Dyson's model, is independent of the macro-position $\theta$, whereas the $N$-particle SDEs depend on $\theta$ and are different from the ISDE in the limit whenever $\theta \neq 0$.
\end{abstract}

Keywords Gaussian unitary ensembles · Dyson's model · Bulk scaling limit · Interacting Brownian motion · Infinite-dimensional stochastic differential equation

Mathematics Subject Classification (2010) 60J60 - 60J70 - 15A52 - 60F17 · 60J65

H.O. is supported in part by JSPS KAKENHI Grant Nos. 16K13764, 16H02149, 16H06338, and KIBAN-A 24244010. Y.K. is supported by Grant-in-Aid for JSPS Research Fellowships (Grant No. 15J03091).

$\bowtie \quad$ Hirofumi Osada

osada@math.kyushu-u.ac.jp

Yosuke Kawamoto

y-kawamoto@math.kyushu-u.ac.jp

1 Faculty of Mathematics, Kyushu University, Fukuoka 819-0395, Japan 


\section{Introduction}

Gaussian unitary ensembles (GUE) are Gaussian ensembles defined on the space of random matrices $M^{N}(N \in \mathbb{N})$ with independent random variables, the matrices of which are Hermitian. By definition, $M^{N}=\left[M_{i, j}^{N}\right]_{i, j=1}^{N}$ is then an $N \times N$ matrix having the form

$$
M_{i, j}^{N}= \begin{cases}\xi_{i} & \text { if } i=j \\ \tau_{i, j} / \sqrt{2}+\sqrt{-1} \zeta_{i, j} / \sqrt{2} & \text { if } i<j\end{cases}
$$

where $\left\{\xi_{i}, \tau_{i, j}, \zeta_{i, j}\right\}_{i<j}^{\infty}$ are i.i.d. Gaussian random variables with mean zero and half variance. Then, the eigenvalues $\lambda_{1}, \ldots, \lambda_{N}$ of $M^{N}$ are real and have distribution $\check{\mu}^{N}$ such that

$$
\check{\mu}^{N}\left(\mathrm{~d} \mathbf{x}_{N}\right)=\frac{1}{Z^{N}} \prod_{i<j}^{N}\left|x_{i}-x_{j}\right|^{2} \prod_{k=1}^{N} e^{-\left|x_{k}\right|^{2}} \mathrm{~d} \mathbf{x}_{N},
$$

where $\mathbf{x}_{N}=\left(x_{1}, \ldots, x_{N}\right) \in \mathbb{R}^{N}$ and $Z^{N}$ is a normalizing constant [1]. Wigner's celebrated semicircle law asserts that their empirical distributions converge in distribution to a semicircle distribution:

$$
\lim _{N \rightarrow \infty} \frac{1}{N}\left\{\delta_{\lambda_{1} / \sqrt{N}}+\cdots+\delta_{\lambda_{N / \sqrt{N}}}\right\}=\frac{1}{\pi} 1_{(-\sqrt{2}, \sqrt{2})}(x) \sqrt{2-x^{2}} \mathrm{~d} x .
$$

One may regard this convergence as a law of large numbers because the limit distribution is a non-random probability measure.

We consider the scaling of the next order in such a way that the distribution is supported on the set of configurations. That is, let $\theta$ be the position of the macroscale given by

$$
-\sqrt{2}<\theta<\sqrt{2}
$$

and take the scaling $x \mapsto y$ such that

$$
x=\frac{y}{\sqrt{N}}+\theta \sqrt{N} .
$$

Let $\mu_{\theta}^{N}$ be the point process for which the labeled density $\mathbf{m}_{\theta}^{N} \mathrm{~d} \mathbf{x}_{N}$ is given by

$$
\mathbf{m}_{\theta}^{N}\left(\mathbf{x}_{N}\right)=\frac{1}{Z^{N}} \prod_{i<j}^{N}\left|x_{i}-x_{j}\right|^{2} \prod_{k=1}^{N} \mathrm{e}^{-\left|x_{k}+\theta N\right|^{2} / N} .
$$


The position $\theta$ in (1.2) is called the bulk and the scaling in (1.3) the bulk scaling (of the point processes). It is well known that the rescaled point processes $\mu_{\theta}^{N}$ satisfy

$$
\lim _{N \rightarrow \infty} \mu_{\theta}^{N}=\mu_{\theta} \quad \text { in distribution, }
$$

where $\mu_{\theta}$ is the determinantal point process with sine kernel $\mathrm{K}_{\theta}$ :

$$
\mathrm{K}_{\theta}(x, y)=\frac{\sin \left\{\sqrt{2-\theta^{2}}(x-y)\right\}}{\pi(x-y)}
$$

By definition, $\mu_{\theta}$ is the point process on $\mathbb{R}$ for which the $m$-point correlation function $\rho_{\theta}^{m}$ with respect to the Lebesgue measure is given by

$$
\rho_{\theta}^{m}\left(x_{1}, \ldots, x_{m}\right)=\operatorname{det}\left[\mathrm{K}_{\theta}\left(x_{i}, x_{j}\right)\right]_{i, j=1}^{m} .
$$

We hence see that the limit is universal in the sense that it is the $\mathrm{Sine}_{2}$ point process and independent of the macro-position $\theta$ up to the dilation of determinantal kernels $\mathrm{K}_{\theta}$. This may be regarded as a first step of the universality of the Sine 2 point process, which has been extensively studied for general inverse temperature $\beta$ and a wide class of free potentials (see [2] and references therein).

Once a static universality is established, then it is natural to enquire of its dynamical counterpart. Indeed, we shall prove the dynamical version of (1.5) and present a phenomenon called stochastic differential equation (SDE) gaps for $\theta \neq 0$.

Two natural $N$-particle dynamics are known for GUE. One is Dyson's Brownian motion corresponding to time-inhomogeneous $N$-particle dynamics given by the time evolution of eigenvalues of time-dependent Hermitian random matrices $\mathcal{M}^{N}(t)$ for which the coefficients are Brownian motions $B_{t}^{i, j}[10]$.

The other is a diffusion process $\mathbf{X}^{\theta, N}=\left(X^{\theta, N, i}\right)_{i=1}^{N}=\left\{\left(X_{t}^{\theta, N, i}\right)_{i=1}^{N}\right\}_{t}$ given by the $\mathrm{SDE}$ such that for $1 \leq i \leq N$

$$
\mathrm{d} X_{t}^{\theta, N, i}=\mathrm{d} B_{t}^{i}+\sum_{j \neq i}^{N} \frac{1}{X_{t}^{\theta, N, i}-X_{t}^{\theta, N, j}} \mathrm{~d} t-\frac{1}{N} X_{t}^{\theta, N, i} \mathrm{~d} t-\theta \mathrm{d} t
$$

which has a unique strong solution for $\mathbf{X}_{0}^{\theta, N} \in \mathbb{R}^{N} \backslash \mathcal{N}$ and $\mathbf{X}^{\theta, N}$ never hits $\mathcal{N}$, where $\mathcal{N}=\left\{\mathbf{x}=\left(x_{k}\right)_{k=1}^{N} ; x_{i}=x_{j}\right.$ for some $\left.i \neq j\right\}[4]$.

The derivation of (1.6) is as follows: Let $\breve{\mu}_{\theta}^{N}\left(\mathrm{~d} \mathbf{x}_{N}\right)=\mathbf{m}_{\theta}^{N}\left(\mathbf{x}_{N}\right) \mathrm{d} \mathbf{x}_{N}$ be the labeled symmetric distribution of $\mu_{\theta}^{N}$. Consider a Dirichlet form on $L^{2}\left(\mathbb{R}^{N}, \breve{\mu}_{\theta}^{N}\right)$ such that

$$
\mathcal{E}^{\check{\mu}_{\theta}^{N}}(f, g)=\int_{\mathbb{R}^{N}} \frac{1}{2} \sum_{i=1}^{N} \frac{\partial f}{\partial x_{i}} \frac{\partial g}{\partial x_{i}} \breve{\mu}_{\theta}^{N}\left(\mathrm{~d} \mathbf{x}_{N}\right)
$$


Then, using (1.4) and integration by parts, we specify the generator $-A^{N}$ of $\mathcal{E}^{\breve{\mu}_{\theta}^{N}}$ on $L^{2}\left(\mathbb{R}^{N}, \check{\mu}_{\theta}^{N}\right)$ such that

$$
A^{N}=\frac{1}{2} \Delta+\sum_{i=1}^{N}\left\{\sum_{j \neq i}^{N} \frac{1}{x_{i}-x_{j}}\right\} \frac{\partial}{\partial x_{i}}-\sum_{i=1}^{N}\left\{\frac{x_{i}}{N}+\theta\right\} \frac{\partial}{\partial x_{i}} .
$$

From this, we deduce that the associated diffusion $\mathbf{X}^{\theta, N}$ is given by (1.6).

Taking the limit $N \rightarrow \infty$ in (1.6), we intuitively obtain the infinite-dimensional SDE (ISDE) of $\mathbf{X}^{\theta}=\left(X^{\theta, i}\right)_{i \in \mathbb{N}}$ such that

$$
\mathrm{d} X_{t}^{\theta, i}=\mathrm{d} B_{t}^{i}+\sum_{j \neq i}^{\infty} \frac{1}{X_{t}^{\theta, i}-X_{t}^{\theta, j}} \mathrm{~d} t-\theta \mathrm{d} t
$$

which was introduced in [22] with $\theta=0$. For each $\theta$, we have a unique, strong solution $\mathbf{X}^{\theta}$ of (1.7) such that $\mathbf{X}_{0}^{\theta}=\mathbf{s}$ for $\mu_{\theta} \circ \mathfrak{l}^{-1}$-a.s. $\mathbf{s}$, where $\mathfrak{l}$ is a labeling map. Although only the $\theta=0$ ISDE of $\mathbf{X}^{0}=: \mathbf{X}=\left(X^{i}\right)_{i \in \mathbb{N}}$ is studied in [17,23], the general $\theta \neq 0$ ISDE is nevertheless follows easily using the transformation

$$
X_{t}^{\theta, i}=X_{t}^{i}-\theta t
$$

Let $\mathbf{X}_{t}^{\theta}=\sum_{i} \delta_{X_{t}^{\theta, i}}$ be the associated delabeled process. Then, $\mathbf{X}^{\theta}=\left\{\mathbf{X}_{t}^{\theta}\right\}$ takes $\mu_{\theta}$ as an invariant probability measure and is not $\mu_{\theta}$-symmetric for $\theta \neq 0$.

The precise meaning of the drift term in (1.7) is the substitution of $\mathbf{X}_{t}^{\theta}=\left(X_{t}^{\theta, i}\right)_{i \in \mathbb{N}}$ for the function $b(x, \mathrm{y})$ given by the conditional sum

$$
b(x, \mathrm{y})=\lim _{r \rightarrow \infty}\left\{\sum_{\left|x-y_{i}\right|<r} \frac{1}{x-y_{i}}\right\}-\theta \quad \text { in } L_{\mathrm{loc}}^{1}\left(\mu_{\theta}^{[1]}\right),
$$

where $\mathrm{y}=\sum_{i} \delta_{y_{i}}$ and $\mu_{\theta}^{[1]}$ is the one-Campbell measure of $\mu_{\theta}$ (see (2.1)). We do this in such a way that $b\left(X_{t}^{\theta, i}, \sum_{j \neq i} \delta_{X_{t}^{\theta, j}}\right)$. Because $\mu_{\theta}$ is translation invariant, it can be easily checked that (1.8) is equivalent to (1.9):

$$
b(x, \mathrm{y})=\lim _{r \rightarrow \infty}\left\{\sum_{\left|y_{i}\right|<r} \frac{1}{x-y_{i}}\right\}-\theta \quad \text { in } L_{\mathrm{loc}}^{1}\left(\mu_{\theta}^{[1]}\right) .
$$

Let $\mathfrak{l}_{N}$ and $\mathfrak{l}$ be labeling maps. We denote by $\mathfrak{l}_{N, m}$ and $\mathfrak{l}_{m}$ the first $m$-components of $\mathfrak{l}_{N}$ and $\mathfrak{l}$, respectively. We assume that, for each $m \in \mathbb{N}$,

$$
\lim _{N \rightarrow \infty} \mu_{\theta}^{N} \circ \mathfrak{l}_{N, m}^{-1}=\mu_{\theta} \circ \mathfrak{l}_{m}^{-1} \text { weakly . }
$$


Let $\mathbf{X}^{\theta, N}=\left(X^{\theta, N, i}\right)_{i=1}^{N}$ and $\mathbf{X}=\left(X^{i}\right)_{i \in \mathbb{N}}$ be solutions of SDEs (1.6) and (1.11), respectively, such that

$$
\begin{gathered}
\mathrm{d} X_{t}^{\theta, N, i}=\mathrm{d} B_{t}^{i}+\sum_{j \neq i}^{N} \frac{1}{X_{t}^{\theta, N, i}-X_{t}^{\theta, N, j}} \mathrm{~d} t-\frac{1}{N} X_{t}^{\theta, N, i} \mathrm{~d} t-\theta \mathrm{d} t, \\
\mathrm{~d} X_{t}^{i}=\mathrm{d} B_{t}^{i}+\lim _{r \rightarrow \infty} \sum_{j \neq i,\left|X_{t}^{i}-X_{t}^{j}\right|<r}^{\infty} \frac{1}{X_{t}^{i}-X_{t}^{j}} \mathrm{~d} t .
\end{gathered}
$$

We now state the first main result of the present paper.

Theorem 1.1 Assume (1.2) and (1.10). Assume that $\boldsymbol{X}_{0}^{\theta, N}=\mu_{\theta}^{N} \circ \mathfrak{l}_{N}^{-1}$ in distribution and $\boldsymbol{X}_{0}=\mu_{\theta} \circ \mathfrak{l}^{-1}$ in distribution. Then, for each $m \in \mathbb{N}$,

$$
\lim _{N \rightarrow \infty}\left(X^{\theta, N, 1}, X^{\theta, N, 2}, \ldots, X^{\theta, N, m}\right)=\left(X^{1}, X^{2}, \ldots, X^{m}\right)
$$

weakly in $C\left([0, \infty), \mathbb{R}^{m}\right)$. In particular, the limit $\boldsymbol{X}=\left(X^{i}\right)_{i \in \mathbb{N}}$ does not satisfy (1.7) for any $\theta$ other than $\theta=0$.

We next consider non-reversible initial distributions. Let $\mathbf{X}^{N}=\left(X^{N, i}\right)_{i=1}^{N}$ and $\mathbf{Y}^{\theta}=\left(Y^{\theta, i}\right)_{i \in \mathbb{N}}$ be solutions of (1.13) and (1.14), respectively, such that

$$
\begin{aligned}
\mathrm{d} X_{t}^{N, i} & =\mathrm{d} B_{t}^{i}+\sum_{j \neq i}^{N} \frac{1}{X_{t}^{N, i}-X_{t}^{N, j}} \mathrm{~d} t-\frac{1}{N} X_{t}^{N, i} \mathrm{~d} t, \\
\mathrm{~d} Y_{t}^{\theta, i} & =\mathrm{d} B_{t}^{i}+\lim _{r \rightarrow \infty} \sum_{j \neq i,\left|Y_{t}^{\theta, i}-Y_{t}^{\theta, j}\right|<r}^{\infty} \frac{1}{Y_{t}^{\theta, i}-Y_{t}^{\theta, j}} \mathrm{~d} t+\theta \mathrm{d} t .
\end{aligned}
$$

Note that $\mathbf{X}^{N}=\mathbf{X}^{0, N}$ and that $\mathbf{X}^{N}$ is not reversible with respect to $\mu_{\theta}^{N} \circ \mathfrak{l}_{N}^{-1}$ for any $\theta \neq 0$. We remark that the delabeld process $\mathbf{Y}^{\theta}=\left\{\sum_{i \in \mathbb{N}} \delta_{Y_{t}^{\theta, i}}\right\}$ of $\mathbf{Y}^{\theta}$ has invariant probability measure $\mu_{\theta}$ and is not symmetric with respect to $\mu_{\theta}$ for $\theta \neq 0$. We state the second main theorem.

Theorem 1.2 Assume (1.2) and (1.10). Assume that $\boldsymbol{X}_{0}^{N}=\mu_{\theta}^{N} \circ \mathfrak{l}_{N}^{-1}$ in distribution and $\boldsymbol{Y}_{0}^{\theta}=\mu_{\theta} \circ \mathfrak{l}^{-1}$ in distribution. Then, for each $m \in \mathbb{N}$

$$
\lim _{N \rightarrow \infty}\left(X^{N, 1}, X^{N, 2}, \ldots, X^{N, m}\right)=\left(Y^{\theta, 1}, Y^{\theta, 2}, \ldots, Y^{\theta, m}\right)
$$

weakly in $C\left([0, \infty), \mathbb{R}^{m}\right)$.

- We refer to the second claim in Theorem 1.1, and (1.15) as the SDE gaps. The convergence in (1.15) of Theorem 1.2 resembles the "Propagation of Chaos" in the 
sense that the limit equation (1.14) depends on the initial distribution, although it is a linear equation. Because the logarithmic potential is by its nature long-ranged, the effect of initial distributions $\mu_{\theta}^{N}$ still remains in the limit ISDE, and the rigidity of the $\mathrm{Sine}_{2}$ point process makes the residual effect a non-random drift term $\theta d t$. There is a result of dynamical universality of Dyson's Brownian motion in [9]. This result is proved in a fairy general situation, but is restricted to finite-particle systems. Our result derives the ISDE from a finite-particle system and is thus regarded as a dynamical universality of Dyson's Brownian motion in infinite dimensions and clarifies that the ISDE of Dyson's Brownian motion in infinite dimensions plays a role of Brownian motion in invariance principle in finite dimensions.

- Let $\mathrm{S}_{\theta}$ be a Borel set such that $\mu_{\theta}\left(\mathrm{S}_{\theta}\right)=1$, where $-\sqrt{2}<\theta<\sqrt{2}$. In [7], the first author proves that one can choose $\boldsymbol{S}_{\theta}$ such that $\boldsymbol{S}_{\theta} \cap \boldsymbol{S}_{\theta^{\prime}}=\emptyset$ if $\theta \neq \theta^{\prime}$ and that for each $s \in S_{\theta}(1.11)$ has a strong solution $\mathbf{X}$ such that $\mathbf{X}=\mathfrak{l}(\mathbf{s})$ and that

$$
\mathrm{X}_{t}:=\sum_{i=1}^{\infty} \delta_{X_{t}^{i}} \in \mathrm{S}_{\theta} \quad \text { for all } t \in[0, \infty)
$$

This implies that the state space of solutions of (1.11) can be decomposed into uncountable disjoint components. We conjecture that the component $\mathrm{S}_{\theta}$ is ergodic for each $\theta \in(-\sqrt{2}, \sqrt{2})$.

- For $\theta=0$, the convergence (1.12) is also proved in [16]. The proof in [16] is algebraic and valid only for dimension $d=1$ and inverse temperature $\beta=2$ with the logarithmic potential. It relies on an explicit calculation of the spacetime correlation functions, the strong Markov property of the stochastic dynamics given by the algebraic construction, the identity of the associated Dirichlet forms constructed by two completely different methods, and the uniqueness of solutions of ISDE (1.7).

Although one may prove (1.10) for $\theta \neq 0$ using the algebraic method in [16], this requires a lot of work as mentioned above. We remark that, as a corollary and an application, Theorem 1.1 proves the weak convergence of finite-dimensional distributions explicitly given by the space-time correlation functions. We refer to $[5,16]$ for the representation of these correlation functions.

- Tsai proves the pathwise uniqueness and the existence of strong solutions of

$$
\mathrm{d} X_{t}^{i}=\mathrm{d} B_{t}^{i}+\frac{\beta}{2} \lim _{r \rightarrow \infty} \sum_{j \neq i,\left|X_{t}^{i}-X_{t}^{j}\right|<r}^{\infty} \frac{1}{X_{t}^{i}-X_{t}^{j}} \mathrm{~d} t \quad(i \in \mathbb{N})
$$

for general $\beta \in[1, \infty)$ in [23]. The proof uses the classical stochastic analysis and crucially depends on a specific monotonicity of SDEs (1.16). For $\beta=1$, 4, we have a good control of the correlation functions as for $\beta=2$. Hence, our method can be applied to $\beta=1,4$ and the same result as for $\beta=2$ in Theorem 1.1 holds. We shall return to this point in future.

It would be an interesting problem to apply Tsai's method to the present problem. One may obtain a convergence at the non-equilibrium level. The difficulty is, 
however, Tsai's method crucially depends on the translation invariance of the stationary measure. As a result, it seems difficult at present to apply it to solve the ISDE for the Airy interacting Brownian motion. It is thus not necessary obvious that Tsai's method is applicable for $\theta \neq 0$ because of lack of the translation invariance.

The key point of the proof of Theorem 1.1 is to prove the convergence of the drift coefficient $b^{N}(x, \mathrm{y})$ of the $N$-particle system to the drift coefficient $b(x, \mathrm{y})$ of the limit ISDE even if $\theta \neq 0$. That is, as $N \rightarrow \infty$,

$$
b^{N}(x, \mathrm{y})=\left\{\sum_{i=1}^{N} \frac{1}{x-y_{i}}\right\}-\theta \Longrightarrow b(x, \mathrm{y})=\lim _{r \rightarrow \infty}\left\{\sum_{\left|y_{i}\right|<r} \frac{1}{x-y_{i}}\right\}
$$

Note that support of the coefficients $b^{N}(x, \mathrm{y})$ and $b(x, \mathrm{y})$ are mutually disjoint and that the sum in $b^{N}$ is not neutral for any $\theta \neq 0$. We shall prove uniform bounds of the tail of the coefficients using fine estimates of the correlation functions and cancel out the deviation of the sum in $b^{N}$ with $\theta$. Because of rigidity of the Sine 2 point process, we justify this cancelation not only for static but also dynamical instances.

The organization of the paper is as follows: In Sect. 2, we prepare general theories for interacting Brownian motion in infinite dimensions. In Sect. 3, we quote estimates for the oscillator wave functions and determinantal kernels. In Sect. 4, we prove key estimates (2.21)-(2.24). In Sect. 5, we complete the proof of Theorem 1.1. In Sect. 6, we prove Theorem 1.2.

\section{Preliminaries from General Theory}

In this section, we present the general theory described in $[8,12,13,17]$ in a reduced form sufficient for the current purpose. In particular, we take the space where particles move in $\mathbb{R}$ rather than $\mathbb{R}^{d}$ as in the cited articles.

\section{1 $\mu$-Reversible Diffusions}

Let $S_{r}=\{s \in \mathbb{R} ;|s|<r\}$. The configuration space $\mathrm{S}$ over $\mathbb{R}$ is a Polish space equipped with the vague topology such that

$$
\mathrm{S}=\left\{\mathrm{s}=\sum_{i} \delta_{s_{i}} ; \mathrm{s}\left(S_{r}\right)<\infty \text { for all } r \in \mathbb{N}\right\}
$$

Each element $S \in S$ is called a configuration regarded as countable delabeled particles. A probability measure $\mu$ on $(\mathrm{S}, \mathcal{B}(\mathrm{S})$ ) is called a point process (a random point field). 
A locally integrable symmetric function $\rho^{n}: \mathbb{R}^{n} \rightarrow[0, \infty)$ is called the $n$-point correlation function of $\mu$ with respect to the Lebesgue measure if $\rho^{n}$ satisfies

$$
\int_{A_{1}^{k_{1}} \times \cdots \times A_{m}^{k_{m}}} \rho^{n}\left(s_{1}, \ldots, s_{n}\right) d \mathbf{s}_{n}=\int_{\mathbf{S}} \prod_{i=1}^{m} \frac{\mathbf{s}\left(A_{i}\right) !}{\left(\mathbf{s}\left(A_{i}\right)-k_{i}\right) !} \mu(d \mathbf{s})
$$

for any sequence of disjoint bounded measurable subsets $A_{1}, \ldots, A_{m} \subset \mathbb{R}$ and a sequence of natural numbers $k_{1}, \ldots, k_{m}$ satisfying $k_{1}+\cdots+k_{m}=n$. Here, we assume that $\mathrm{s}\left(A_{i}\right) ! /\left(\mathrm{s}\left(A_{i}\right)-k_{i}\right) !=0$ for $\mathrm{s}\left(A_{i}\right)-k_{i}<0$.

Let $\Phi: \mathbb{R} \rightarrow \mathbb{R}$ and $\Psi: \mathbb{R}^{2} \rightarrow \mathbb{R} \cup\{\infty\}$ be measurable functions called free and interaction potentials, respectively. Let $\mathcal{H}_{r}$ be the Hamiltonian on $S_{r}$ given by

$$
\mathcal{H}_{r}(\mathbf{x})=\sum_{x_{i} \in S_{r}} \Phi\left(x_{i}\right)+\sum_{j \neq k, x_{j}, x_{k} \in S_{r}} \Psi\left(x_{j}, x_{k}\right) \quad \text { for } \mathbf{x}=\sum_{i} \delta_{x_{i}}
$$

For each $m, r \in \mathbb{N}$ and $\mu$-a.s. $\xi \in \mathrm{S}$, let $\mu_{r, \xi}^{m}$ denote the regular conditional probability such that

$$
\mu_{r, \xi}^{m}=\mu\left(\pi_{S_{r}}(\mathbf{x}) \in \cdot \mid \pi_{S_{r}^{c}}(\mathbf{x})=\pi_{S_{r}^{c}}(\xi), \mathbf{x}\left(S_{r}\right)=m\right) .
$$

Here, for a subset $A$, we set $\pi_{A}: \mathrm{S} \rightarrow \mathrm{S}$ by $\pi_{A}(\mathrm{~s})=\mathrm{s}(\cdot \cap A)$.

Let $\Lambda_{r}$ denote the Poisson point process with intensity being a Lebesgue measure on $S_{r}$. We set $\Lambda_{r}^{m}(\cdot)=\Lambda_{r}\left(\cdot \cap \mathrm{S}_{r}^{m}\right)$, where $\mathrm{S}_{r}^{m}=\left\{\mathrm{s} \in \mathrm{S} ; \mathrm{s}\left(S_{r}\right)=m\right\}$.

Definition 1 ([13], [14]) A point process $\mu$ is said to be a $(\Phi, \Psi)$-quasi-Gibbs measure if its regular conditional probabilities $\mu_{r, \xi}^{m}$ satisfy, for any $r, m \in \mathbb{N}$ and $\mu$-a.s. $\xi$,

$$
c_{1}^{-1} e^{-\mathcal{H}_{r}(\mathbf{x})} \Lambda_{r}^{m}(\mathrm{dx}) \leq \mu_{r, \xi}^{m}(\mathrm{~d} \mathbf{x}) \leq c_{1} e^{-\mathcal{H}_{r}(\mathbf{x})} \Lambda_{r}^{m}(\mathrm{~d} \mathbf{x})
$$

Here, $c_{1}$ is a positive constant depending on $r, m, \xi$.

The significance of the quasi-Gibbs property is to guarantee the existence of $\mu$ reversible diffusion process $\left\{P_{\mathrm{S}}\right\}$ on $\mathrm{S}$ given by the natural Dirichlet form associated with $\mu$, in analogy with distorted Brownian motion in finite dimensions.

To introduce the Dirichlet form, we provide some notations. We say a function $f$ on $\mathrm{S}$ is local if $f$ is $\sigma\left[\pi_{K}\right]$-measurable for some compact set $K$ in $\mathbb{R}$. For a local function $f$ on $\mathrm{S}$, we say $f$ is smooth if $\breve{f}$ is smooth, where $\breve{f}\left(x_{1}, \ldots\right)$ is the symmetric function such that $\breve{f}\left(x_{1}, \ldots\right)=f(\mathbf{x})$ for $\mathbf{x}=\sum_{i} \delta_{x_{i}}$. Let $\mathcal{D}$ 。 be the set of all bounded, locally smooth functions on $\mathrm{S}$.

Let $\mathbb{D}$ be the standard square field on $\mathrm{S}$ such that for $f, g \in \mathcal{D}_{\circ}$ and $\mathrm{s}=\sum_{i} \delta_{s_{i}}$

$$
\mathbb{D}[f, g](\mathbf{s})=\frac{1}{2}\left\{\sum_{i}\left(\nabla_{i} \check{f}\right)\left(\nabla_{i} \check{g}\right)\right\}
$$


We write $\mathbf{s}=\left(s_{i}\right)_{i}$. Because the function $\sum_{i}\left(\nabla_{i} \check{f}\right)(\mathbf{s})\left(\nabla_{i} \check{g}\right)(\mathbf{s})$ is symmetric in $\mathbf{s}=$ $\left(s_{i}\right)_{i}$, we regard it as a function of s. We set $L^{2}(\mu)=L^{2}(\mathrm{~S}, \mu)$ and let

$$
\mathcal{E}^{\mu}(f, g)=\int_{\mathbf{S}} \mathbb{D}[f, g](\mathbf{s}) \mu(d \mathbf{s}), \quad \mathcal{D}_{\circ}^{\mu}=\left\{f \in \mathcal{D}_{\circ} \cap L^{2}(\mu) ; \mathcal{E}^{\mu}(f, f)<\infty\right\}
$$

We quote:

Lemma 1 ([13]) Assume that $\mu$ is a $(\Phi, \Psi)$-quasi-Gibbs measure with upper semicontinuous $(\Phi, \Psi)$. Assume that the correlation functions $\left\{\rho^{n}\right\}$ are locally bounded for all $n \in \mathbb{N}$. Then, $\left(\mathcal{E}^{\mu}, \mathcal{D}_{\circ}^{\mu}\right)$ is closable on $L^{2}(\mu)$. Furthermore, there exists a $\mu$ reversible diffusion process $\left\{P_{\mathrm{S}}\right\}$ associate with the Dirichlet form $\left(\mathcal{E}^{\mu}, \mathcal{D}^{\mu}\right)$ on $L^{2}(\mu)$. Here, $\left(\mathcal{E}^{\mu}, \mathcal{D}^{\mu}\right)$ is the closure of $\left(\mathcal{E}^{\mu}, \mathcal{D}_{\circ}^{\mu}\right)$ on $L^{2}(\mu)$.

\subsection{Infinite-Dimensional SDEs}

Suppose that diffusion $\left\{P_{\mathrm{S}}\right\}$ in Lemma 1 is collision-free and that each tagged particle does not explode. Then, we can construct labeled dynamics $\mathbf{X}=\left(X^{i}\right)_{i \in \mathbb{Z}}$ by introducing the initial labeling $\mathfrak{l}=\left(\mathfrak{l}_{i}\right)_{i \in \mathbb{Z}}$ such that

$$
\mathbf{X}_{0}=\mathfrak{l}\left(\mathbf{X}_{0}\right)
$$

Indeed, once the label $\mathfrak{l}$ is given at time zero, then each particle retains the tag for all time because of the collision-free and explosion-free property.

To specify the ISDEs satisfied by $\mathbf{X}$ above, we introduce the notion of the logarithmic derivative of $\mu$, which was introduced in [12].

A point process $\mu_{x}$ is called the reduced Palm measure of $\mu$ conditioned at $x \in \mathbb{R}$ if $\mu_{x}$ is the regular conditional probability defined as

$$
\mu_{x}=\mu\left(\cdot-\delta_{x} \mid \mathbf{s}(\{x\}) \geq 1\right) .
$$

A Radon measure $\mu^{[1]}$ on $\mathbb{R} \times \mathrm{S}$ is called the 1-Campbell measure of $\mu$ if

$$
\mu^{[1]}(\mathrm{d} x \mathrm{ds})=\rho^{1}(x) \mu_{x}(\mathrm{~d} \mathbf{s}) \mathrm{d} x .
$$

We write $f \in L_{\text {loc }}^{p}\left(\mu^{[1]}\right)$ if $f \in L^{p}\left(S_{r} \times \mathrm{S}, \mu^{[1]}\right)$ for all $r \in \mathbb{N}$.

Definition 2 A $\mathbb{R}$-valued function $\mathrm{d}^{\mu} \in L_{\text {loc }}^{1}\left(\mu^{[1]}\right)$ is called the logarithmic derivative of $\mu$ if, for all $\varphi \in C_{0}^{\infty}(\mathbb{R}) \otimes \mathcal{D}_{\circ}$,

$$
\int_{\mathbb{R} \times \mathrm{S}} \mathrm{d}^{\mu}(x, \mathrm{y}) \varphi(x, \mathrm{y}) \mu^{[1]}(\mathrm{d} x \mathrm{dy})=-\int_{\mathbb{R} \times \mathrm{S}} \nabla_{x} \varphi(x, \mathrm{y}) \mu^{[1]}(\mathrm{d} x \mathrm{dy}) .
$$

Under these assumptions, we obtain the following: 
Lemma 2 ([12]) Assume that $\boldsymbol{X}=\left(X^{i}\right)_{i \in \mathbb{N}}$ is the collision-free and explosion-free. Then, $\boldsymbol{X}$ is a solution of the following ISDE:

$$
\mathrm{d} X_{t}^{i}=\mathrm{d} B_{t}^{i}+\frac{1}{2} d^{\mu}\left(X_{t}^{i}, X_{t}^{\diamond i}\right) \mathrm{d} t \quad(i \in \mathbb{N})
$$

with initial condition $\boldsymbol{X}_{0}=\boldsymbol{s}$ for $\mu \circ \mathfrak{l}^{-1}$-a.s. s, where $X_{t}^{\triangleright i}=\sum_{j \neq i}^{\infty} \delta_{X_{t}^{j}}$.

\subsection{Finite-Particle Approximations}

Let $\mu$ be a point process with correlation functions $\left\{\rho^{n}\right\}_{n \in \mathbb{N}}$. Let $\left\{\mu^{N}\right\}_{N \in \mathbb{N}}$ be a sequence of point processes on $\mathbb{R}$ such that $\mu^{N}(\{\mathrm{~s}(\mathbb{R})=N\})=1$. We assume: (A1) Each $\mu^{N}$ has correlation functions $\left\{\rho^{N, n}\right\}_{n \in \mathbb{N}}$ satisfying, for each $r \in \mathbb{N}$,

$$
\begin{aligned}
& \lim _{N \rightarrow \infty} \rho^{N, n}(\mathbf{x})=\rho^{n}(\mathbf{x}) \quad \text { uniformly on } S_{r}^{n} \text { for each } n \in \mathbb{N}, \\
& \sup _{N \in \mathbb{N}} \sup _{\mathbf{x} \in S_{r}^{n}} \rho^{N, n}(\mathbf{x}) \leq c_{2}^{n} n^{c_{3} n},
\end{aligned}
$$

where $0<c_{2}(r)<\infty$ and $0<c_{3}(r)<1$ are constants independent of $n \in \mathbb{N}$.

It is known that (2.3) and (2.4) imply the weak convergence of $\left\{\mu^{N}\right\}$ to $\mu[13$, Lemma A.1]. As in Sect. 1, let $\mathfrak{l}$ and $\mathfrak{l}_{N}$ be labels of $\mu$ and $\mu^{N}$, respectively. We assume:

(A2) For each $m \in \mathbb{N}$,

$$
\lim _{N \rightarrow \infty} \mu^{N} \circ \mathfrak{l}_{N, m}^{-1}=\mu \circ \mathfrak{l}_{m}^{-1} \quad \text { weakly in } \mathbb{R}^{m} .
$$

We shall later take $\mu^{N} \circ \mathfrak{l}_{N}^{-1}$ as an initial distribution of labeled finite-particle system. Therefore, (A2) means the convergence of the initial distribution of the labeled dynamics.

For a labeled process $\mathbf{X}^{N}=\left(X^{N, i}\right)_{i=1}^{N}$, where $N \in \mathbb{N}$, we set

$$
\mathrm{X}_{t}^{N, \diamond i}=\sum_{j \neq i}^{N} \delta_{X_{t}^{N, j}}
$$

where $\mathrm{X}_{t}^{N, \diamond i}$ denotes the zero measure for $N=1$. Let $\mathrm{b}^{N}, \mathrm{~b}: \mathbb{R} \times \mathrm{S} \rightarrow \mathbb{R}$ be measurable functions. We introduce the finite-dimensional SDE of $\mathbf{X}^{N}=\left(X^{N, i}\right)_{i=1}^{N}$ with these coefficients such that for $1 \leq i \leq N$

$$
\mathrm{d} X_{t}^{N, i}=\mathrm{d} B_{t}^{i}+\mathrm{b}^{N}\left(X_{t}^{N, i}, \mathrm{X}_{t}^{N, \diamond i}\right) \mathrm{d} t
$$

We assume:

(A3) SDE (2.5) with initial condition $\mathbf{X}_{0}^{N}=\mathbf{s}$ has a unique solution for $\mu^{N} \circ \mathfrak{l}_{N}^{-1}$-a.s. s for each $N$. This solution does not explode. 
Let $u, u^{N}, w: \mathbb{R} \rightarrow \mathbb{R}$ and $g: \mathbb{R}^{2} \rightarrow \mathbb{R}$ be measurable functions. We set

$$
\begin{aligned}
& \mathrm{g}_{r}(x, \mathrm{y})=\sum_{i} \chi_{r}\left(x-y_{i}\right) g\left(x, y_{i}\right), \\
& w_{r}(x, \mathrm{y})=\sum_{i}\left(1-\chi_{r}\left(x-y_{i}\right)\right) g\left(x, y_{i}\right),
\end{aligned}
$$

where $\mathrm{y}=\sum_{i} \delta_{y_{i}}$ and $\chi_{r} \in C_{0}^{\infty}(\mathbb{R})$ is a cut-off function such that $0 \leq \chi_{r} \leq 1$, $\chi_{r}(x)=0$ for $|x| \geq r+1$, and $\chi_{r}(x)=1$ for $|x| \leq r$. We assume the following. (A4) Each $\mu^{N}$ has a logarithmic derivative $\mathrm{d}^{N}$ such that

$$
\mathrm{d}^{N}(x, \mathrm{y})=u^{N}(x)+\mathrm{g}_{r}(x, \mathrm{y})+w_{r}(x, \mathrm{y}) .
$$

Furthermore, we assume that

(1) $u^{N}$ are in $C^{1}(\mathbb{R})$. Furthermore, $u^{N}$ and $\nabla u^{N}$ converge uniformly to $u$ and $\nabla u$, respectively, on each compact set in $\mathbb{R}$.

(2) $g \in C^{1}\left(\mathbb{R}^{2} \cap\{x \neq y\}\right)$. There exists a $\hat{p}>1$ such that, for each $R \in \mathbb{N}$,

$$
\lim _{\mathrm{p} \rightarrow \infty} \limsup _{N \rightarrow \infty} \int_{x \in S_{R},|x-y| \leq 2^{-\mathrm{p}}} \chi_{r}(x-y)|g(x, y)|^{\hat{p}} \rho_{x}^{N, 1}(y) \mathrm{d} x \mathrm{~d} y=0,
$$

where $\rho_{x}^{N, 1}$ is a one-correlation function of the reduced Palm measure $\mu_{x}^{N}$.

(3) There exists a continuous function $w: \mathbb{R} \rightarrow \mathbb{R}$ such that for each $R \in \mathbb{N}$

$$
\lim _{r \rightarrow \infty} \limsup _{N \rightarrow \infty} \int_{S_{R} \times \mathrm{S}}\left|w_{r}(x, \mathrm{y})-w(x)\right|{ }^{\hat{p}} \mathrm{~d} \mu^{N,[1]}=0 .
$$

Let $p$ be such that $1<p<\hat{p}$. Assume (A1) and (A4). Then, [12, Theorem 45] deduces that the logarithmic derivative $\mathrm{d}^{\mu}$ of $\mu$ exists in $L_{\text {loc }}^{p}\left(\mu^{[1]}\right)$ and is given by

$$
\mathrm{d}^{\mu}(x, \mathrm{y})=u(x)+\mathrm{g}(x, \mathrm{y})+w(x)
$$

Here, $\mathrm{g}(x, \mathrm{y})=\lim _{r \rightarrow \infty} \mathrm{g}_{r}(x, \mathrm{y})$ and the convergence of lim $\mathrm{g}_{r}$ takes place in $L_{\text {loc }}^{p}\left(\mu^{[1]}\right)$. Taking (2.11) into account, we introduce the ISDE of $\mathbf{X}=\left(X^{i}\right)_{i \in \mathbb{N}}$ :

$$
\mathrm{d} X_{t}^{i}=\mathrm{d} B_{t}^{i}+\frac{1}{2}\left\{u\left(X_{t}^{i}\right)+\mathrm{g}\left(X_{t}^{i}, \mathbf{X}_{t}^{\diamond i}\right)+w\left(X_{t}^{i}\right)\right\} \mathrm{d} t
$$

Under the assumptions of Lemma 2, ISDE (2.12) with $\mathbf{X}_{0}=\mathbf{s}$ has a solution for $\mu \circ \mathfrak{l}^{-1}$-a.s.s. Moreover, the associated delabeled diffusion $X=\left\{X_{t}\right\}$ is $\mu$-reversible, where $\mathbf{X}_{t}=\sum_{i \in \mathbb{N}} \delta_{X_{t}^{i}}$ for $\mathbf{X}_{t}=\left(X_{t}^{i}\right)_{i \in \mathbb{N}}$. As for uniqueness, we recall the notion of $\mu$-absolute continuity solution introduced in [17].

Let $\mathbf{X}=\left(X^{i}\right)_{i \in \mathbb{N}}$ be a family of solution of (2.12) satisfying $\mathbf{X}_{0}=\mathbf{s}$ for $\mu \circ \mathfrak{l}^{-1}$-a.s. s. Let $\mu_{t}$ be the distribution of the delabeled process $\mathbf{X}_{t}=\sum_{i \in \mathbb{N}} \delta_{X_{t}^{i}}$ at time $t$ with initial distribution $\mu$. That is, $\mu_{t}$ is given by 


$$
\mu_{t}=\int_{\mathrm{S}} P_{\mathrm{S}}\left(\mathrm{X}_{t} \in \cdot\right) \mathrm{d} \mu
$$

We say that $\mathbf{X}$ satisfies the $\mu$-absolute continuity condition if

$$
\mu_{t} \prec \mu \quad \text { for all } t \geq 0 \text {, }
$$

where $\mu_{t} \prec \mu$ means that $\mu_{t}$ is absolutely continuous with respect to $\mu$. If $\mathbf{X}$ is $\mu$-reversible, then (2.13) is satisfied.

We say ISDE (2.12) has $\mu$-uniqueness in law of solutions if $\mathbf{X}$ and $\mathbf{X}^{\prime}$ are solutions with the same initial distributions satisfying the $\mu$-absolute continuity condition, then they are equivalent in law. We assume:

(A5) ISDE (2.12) has $\mu$-uniqueness in law of solutions.

It is proved in [17] that ISDE (2.2) has a strong solution and a solution of (2.2) is pathwise unique for almost sure staring points if, loosely speaking, $\mu$ is tail trivial, the logarithmic derivative $\mathrm{d}^{\mu}$ has a sort of off-diagonal smoothness, and the onecorrelation function has sub-exponential growth at infinity. This results implies $\mu$ uniqueness in law. We refer to Theorems 2.1 in [17] for details. The next result is a special case of [8, Theorem 2.1].

Lemma 3 ([8, Theorem 2.1]) Make the same assumptions in Lemmas 1 and 2. Assume (A1)-(A4). Assume that $\boldsymbol{X}_{0}^{N}=\mu^{N} \circ \mathfrak{l}_{N}^{-1}$ in distribution. Then, $\left\{\boldsymbol{X}^{N}\right\}_{N \in \mathbb{N}}$ is tight in $C\left([0, \infty) ; \mathbb{R}^{\mathbb{N}}\right)$ and each limit point $\boldsymbol{X}$ of $\left\{\boldsymbol{X}^{N}\right\}_{N \in \mathbb{N}}$ is a solution of (2.12) with initial distribution $\mu \circ \mathfrak{l}^{-1}$. If, in addition, we assume (A5), then for any $m \in \mathbb{N}$

$$
\lim _{N \rightarrow \infty}\left(X^{N, 1}, \ldots, X^{N, m}\right)=\left(X^{1}, \ldots, X^{m}\right) .
$$

weakly in $C\left([0, \infty), \mathbb{R}^{m}\right)$. Here, $\boldsymbol{X}^{N}=\left(X^{N, i}\right)_{i=1}^{N}$ and $\boldsymbol{X}=\left(X^{i}\right)_{i \in \mathbb{N}}$ as before.

\subsection{Reduction of Theorem 1.1 to $(2.10)$}

In this subsection, we deduce Theorem 1.1 from Lemma 3 by assuming (2.10). We take $\mu_{\theta}^{N}$ and $\mu_{\theta}$ as in Sect. 1. Then, the logarithmic derivative $\mathrm{d}^{\mu_{\theta}^{N}}$ of $\mu_{\theta}^{N}$ is given by

$$
\mathrm{d}^{\mu_{\theta}^{N}}(x, \mathrm{y})=\sum_{i=1}^{N} \frac{2}{x-y_{i}}-\frac{2 x}{N}-2 \theta,
$$

where $\mathrm{y}=\sum_{i} \delta_{y_{i}}$. From (2.14), we take coefficients in (A4) as follows:

$$
\begin{aligned}
u^{N}(x) & =-\frac{2 x}{N}-2 \theta, \quad u(x)=-2 \theta, \quad w(x)=2 \theta, \\
g(x, y) & =\frac{2}{x-y} .
\end{aligned}
$$

Other functions are given by (2.6) and (2.7). 
Lemma 4 Assume (2.10) holds with $\hat{p}=2$ for the coefficients as above. Then, (1.12) holds.

Proof To prove Lemma 4, we check the assumptions in Lemma 3, that is, the assumptions in Lemma 1, Lemma 2, and (A1)-(A5).

The assumptions in Lemma 1 are proved in [13]. The assumptions in Lemma 2 are checked in [12]. (A1) is well known. (A2) is assumed by (1.10). (A3) is obvious as the interaction is smooth outside the origin, and the capacity of the colliding set $\left\{x_{i}=\right.$ $x_{j}$ for some $\left.i \neq j\right\}$ is zero (see [4,11]). Furthermore, the one-correlation functions are bounded, which guarantees explosion-free of tagged particles. We take functions in (A4) as (2.15) and (2.16). These satisfy (2.8), (2.9), and (1) of (A4). (2.10) is satisfied by assumption. It is known that $\mu_{\theta}$ is tail trivial [15]. Then, (A5) follows from tail triviality of $\mu_{\theta}$ and [17, Theorem 3.1]. All the assumptions in Lemma 3 are thus satisfied and hence yield (1.12).

\subsection{A Sufficient Condition for (2.10)}

The most crucial step to apply Lemma 3 is to check (2.10). Indeed, it only remains to prove (2.10) for Theorem 1.1. We quote then a sufficient condition for (2.10) in terms of correlation functions from [12]. Lemma 6 is a special case of [12, Lemma 53].

Let $\mu_{\theta, x}^{N}$ be the reduced Palm measure of $\mu_{\theta}^{N}$ conditioned at $x$. We denote the supremum norm in $x$ over $S_{R}$ by $\|\cdot\|_{R}$. Let $\mathrm{E}^{\cdot}$ and Var denote the expectation and variance with respect to $\cdot$, respectively.

Lemma 5 Assume $|\theta|<\sqrt{2}$. Let $w_{r}$ be as in (2.7) with $g(x, y)$ given by (2.16). Let $w(x)=2 \theta$ as in (2.15). Then, (2.10) follows from (2.17)-(2.20).

$$
\begin{aligned}
& \lim _{r \rightarrow \infty} \limsup _{N \rightarrow \infty}\left\|\mathrm{E}^{\mu_{\theta}^{N}}\left[w_{r}(x, \mathrm{y})\right]-2 \theta\right\|_{R}=0,
\end{aligned}
$$

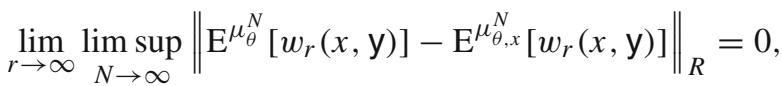

$$
\begin{aligned}
& \lim _{r \rightarrow \infty} \limsup _{N \rightarrow \infty}\left\|\operatorname{Var}^{\mu_{\theta}^{N}}\left[w_{r}(x, \mathrm{y})\right]\right\|_{R}=0 \\
& \lim _{r \rightarrow \infty} \limsup _{N \rightarrow \infty}\left\|\operatorname{Var}^{\mu_{\theta}^{N}}\left[w_{r}(x, \mathrm{y})\right]-\operatorname{Var}^{\mu_{\theta, x}^{N}}\left[w_{r}(x, \mathrm{y})\right]\right\|_{R}=0 .
\end{aligned}
$$

Proof Lemma 5 follows from [12, Lemma 52]. Indeed, (2.17), (2.18), (2.19), and (2.20) in the present paper correspond to (5.4), (5.2), (5.5), and (5.3) in [12], respectively. We note that in [12] we use $1_{S_{r}}(x)$ instead of $\chi_{r}(x)$. This slight modification yields no difficulty.

Multiplying $w_{r}(x, \mathrm{y})$ by a half, we give a sufficient condition of (2.17)-(2.20) in terms of correlation functions. Let $\rho_{\theta, x}^{N, m}$ and $\rho_{\theta}^{N, m}$ be the $m$-point correlation functions of $\mu_{\theta, x}^{N}$ and $\mu_{\theta}^{N}$, respectively. Let

$$
S_{r, \infty}(x)=S_{r *}^{x}=\{y \in \mathbb{R} ; r<|x-y|<\infty\} .
$$


Lemma 6 Assume $|\theta|<\sqrt{2}$. Then, (2.17)-(2.20) follow from (2.21)-(2.24).

$$
\begin{aligned}
& \lim _{r \rightarrow \infty} \limsup _{N \rightarrow \infty}\left\|\int_{S_{r *}^{x}} \frac{\rho_{\theta}^{N, 1}(y)}{x-y} \mathrm{~d} y-\theta\right\|_{R}=0, \\
& \lim _{r \rightarrow \infty} \limsup _{N \rightarrow \infty}\left\|\int_{S_{r *}^{x}} \frac{\rho_{\theta, x}^{N, 1}(y)-\rho_{\theta}^{N, 1}(y)}{x-y} \mathrm{~d} y\right\|_{R}=0, \\
& \lim _{r \rightarrow \infty} \limsup _{N \rightarrow \infty} \| \int_{S_{r *}^{x}} \frac{\rho_{\theta}^{N, 1}(y)}{(x-y)^{2}} \mathrm{~d} y \\
& \quad+\int_{\left(S_{r *}^{x}\right)^{2}} \frac{\rho_{\theta}^{N, 2}(y, z)-\rho_{\theta}^{N, 1}(y) \rho_{\theta}^{N, 1}(z)}{(x-y)(x-z)} \mathrm{d} y \mathrm{~d} z \|_{R}=0,
\end{aligned}
$$

$\lim _{r \rightarrow \infty} \limsup _{N \rightarrow \infty} \| \int_{S_{r *}^{x}} \frac{\rho_{\theta, x}^{N, 1}(y)-\rho_{\theta}^{N, 1}(y)}{(x-y)^{2}} \mathrm{~d} y+$

$\int_{\left(S_{r *}^{x}\right)} \frac{\rho_{\theta, x}^{N, 2}(y, z)-\rho_{\theta, x}^{N, 1}(y) \rho_{\theta, x}^{N, 1}(z)-\left\{\rho_{\theta}^{N, 2}(y, z)-\rho_{\theta}^{N, 1}(y) \rho_{\theta}^{N, 1}(z)\right\}}{(x-y)(x-z)} \mathrm{d} y \mathrm{~d} z \|_{R}=0$.

Proof Lemma 6 follows immediately from a standard calculation of correlation functions and the definitions of $w_{r}$ and $\chi_{r}$.

\section{Subsidiary Estimates}

Keeping Lemma 6 in mind, our task is to prove (2.21)-(2.24). To control the correlation functions in Lemma 6, we prepare in this section estimates of the oscillator wave functions and determinantal kernels. We shall use these estimates in Sect. 4.

\subsection{Oscillator Wave Functions}

Let $H_{n}(x)=(-1)^{n} e^{x^{2}}\left(\frac{d}{d x}\right)^{n} e^{-x^{2}}$ be Hermite polynomials. Let $\psi_{n}(x)$ denote the oscillator wave functions defined by

$$
\psi_{n}(x)=\frac{1}{\sqrt{\sqrt{\pi} 2^{n} n !}} e^{-\frac{x^{2}}{2}} H_{n}(x)
$$

Note that $\left\{\psi_{n}\right\}_{n=0}^{\infty}$ is an orthonormal system; $\int_{\mathbb{R}} \psi_{n}(x) \psi_{m}(x) \mathrm{d} x=\delta_{n m}$.

The following estimates for these oscillator wave functions are essentially due to Plancherel-Rotach [19]. We quote here a version from Katori-Tanemura [6].

Lemma 7 ([6]) Let $C_{n m}^{1}, C_{n m}^{2}$, and $D_{n m}^{1}$ be the constants introduced in [6] (see (A.1) in $[6,572 \mathrm{p}]$ ). Let $l=-1,0,1$ and $N, L \in \mathbb{N}$. Then, we have the following. 
(1) Let $0<\tau \leq \frac{\pi}{2}$. Assume that $N \sin ^{3} \tau \geq C N^{\varepsilon}$ for some $C>0$ and $\varepsilon>0$. Then,

$$
\begin{aligned}
& \psi_{N+l}(\sqrt{2 N} \cos \tau)=\frac{1+\mathcal{O}\left(N^{-1}\right)}{\sqrt{\pi \sin \tau}}\left(\frac{2}{N}\right)^{\frac{1}{4}} \\
& \times\left[\sum_{n=0}^{L-1} \sum_{m=0}^{n} C_{n m}^{1}(N+l, \tau) \sin \left\{\frac{N}{2}(2 \tau-\sin 2 \tau)+D_{n m}^{1}(\tau)-(1+l) \tau\right\}\right. \\
& \left.\quad+\mathcal{O}\left(\frac{1}{N \sin \tau}\right)\right]
\end{aligned}
$$

(2) Let $\tau>0$. Assume that $N \sinh ^{3} \tau \geq C N^{\varepsilon}$ for some $C>0$ and $\varepsilon>0$. Then,

$$
\begin{aligned}
& \psi_{N+l}(\sqrt{2 N} \cosh \tau) \\
& =\frac{1+\mathcal{O}\left(N^{-1}\right)}{\sqrt{2 \pi \sinh \tau}}\left(\frac{1}{2 N}\right)^{\frac{1}{4}} \exp \left[\left(\frac{N+1+l}{2}\right)(2 \tau-\sinh 2 \tau)+(1+l) \tau\right] \\
& \times\left[\sum_{n=0}^{L-1} \sum_{m=0}^{n} C_{n m}^{2}(\tau, N+l)+\mathcal{O}\left(\frac{\cosh ^{3} \tau}{N \sinh \tau}\right)\right]
\end{aligned}
$$

Proof (1) and (2) follow from (5.5) and (5.10) in [6], respectively.

We next quote estimates from $[6,18]$.

\section{Lemma 8 ([6], [18])}

(1) Let $y=\sqrt{2 N} \cos \tau$ with $N \in \mathbb{N}$ and $0<\tau \leq \frac{\pi}{2}$. Assume that $N \sin ^{3} \tau \geq C N^{\varepsilon}$ for some $C>0$ and $\varepsilon>0$. Then,

$$
\sum_{k=0}^{N-1} \psi_{k}(y)^{2}=\frac{1}{\pi} \sqrt{2 N-y^{2}}+\mathcal{O}\left(\frac{\sqrt{N}}{2 N-y^{2}}\right) .
$$

(2) Let $y=\sqrt{2 N} \cosh \tau$ with $N \in \mathbb{N}$ and $\tau>0$. Assume that $N \sinh ^{3} \tau \geq C N^{\varepsilon}$ for some $C>0$ and $\varepsilon>0$. Then,

$$
\sum_{k=0}^{N-1} \psi_{k}(y)^{2}=\mathcal{O}\left(\frac{\sqrt{N}}{y^{2}-2 N}\right)
$$

(3) There is a positive constant $c_{4}$ such that for all $N \in \mathbb{N}$

$$
\sup _{y \in \mathbb{R}}\left|\psi_{N}(y)\right| \leq c_{4} N^{-\frac{1}{12}}
$$


Proof (1) follows from Lemma 5.2 (i) in [6]. (2) follows from Lemma 5.2 (ii) in [6]. From Lemma 6.9 in [18], there exists a constant $c_{4}$ such that

$$
\left|N^{\frac{1}{12}} \psi_{N}\left(2 \sqrt{N}+y N^{-\frac{1}{6}}\right)\right| \leq \frac{c_{4}}{(1 \vee|y|)^{\frac{1}{4}}}, \quad y \in\left[-2 N^{\frac{2}{3}}, \infty\right) .
$$

Hence, we have

$$
\left|\psi_{N}(y)\right| \leq \frac{c_{4}}{N^{\frac{1}{12}}\left(1 \vee\left\{N^{\frac{1}{6}}|y-2 \sqrt{N}|\right\}\right)^{\frac{1}{4}}}, \quad y \in[0, \infty) .
$$

Claim (3.2) is immediate from (3.3) and the well-known property such that $\psi_{N}(y)=$ $\psi_{N}(-y)$ if $N$ is even and that $\psi_{N}(y)=-\psi_{N}(-y)$ if $N$ is odd.

\subsection{Determinantal Kernels of $N$-Particle Systems}

We recall the definition of determinantal point processes. Let $K: \mathbb{R}^{2} \rightarrow \mathbb{C}$ be a measurable kernel. A probability measure $\mu$ on $S$ is called a determinantal point process with kernel $K$ if, for each $n$, its $n$-point correlation function is given by

$$
\rho^{n}\left(x_{1}, \ldots, x_{n}\right)=\operatorname{det}\left[K\left(x_{i}, x_{j}\right)\right]_{i, j=1}^{n} \text {. }
$$

If $K$ is an Hermitian symmetric and of locally trace class such that $0 \leq \operatorname{Spec}(K) \leq 1$, then there exists a unique determinantal point process with kernel $K$ [20,21].

The distribution of the delabeled eigenvalues of GUE associated with (1.1) is a determinantal point process with kernel $\mathrm{K}^{N}$ such that

$$
\mathrm{K}^{N}(x, y)=\sum_{k=0}^{N-1} \psi_{k}(x) \psi_{k}(y)
$$

The Christoffel-Darboux formula and a simple calculation yield the following.

$$
\mathrm{K}^{N}(x, y)=\sqrt{\frac{N}{2}} \frac{\psi_{N}(x) \psi_{N-1}(y)-\psi_{N-1}(x) \psi_{N}(y)}{x-y} .
$$

From the scaling (1.3), $\mu_{\theta}^{N}$ is a determinantal point process with kernel

$$
\mathrm{K}_{\theta}^{N}(x, y)=\frac{1}{\sqrt{N}} \mathrm{~K}^{N}\left(\frac{x+N \theta}{\sqrt{N}}, \frac{y+N \theta}{\sqrt{N}}\right) .
$$

Let $x_{N}=\sqrt{N} x$ and $y_{N}=\sqrt{N} y$. We set

$$
\mathrm{L}^{N}(x, y)=\frac{1}{\sqrt{N}} \mathrm{~K}^{N}\left(x_{N}, y_{N}\right)=\frac{1}{\sqrt{N}} \mathrm{~K}^{N}(\sqrt{N} x, \sqrt{N} y) .
$$


From (3.7) and (3.8), we then clearly see that

$$
\begin{aligned}
& \mathrm{K}_{\theta}^{N}(x, y)=\mathrm{L}^{N}\left(\frac{x}{N}+\theta, \frac{y}{N}+\theta\right), \\
& \mathrm{L}^{N}(x, y)=\mathrm{K}_{\theta}^{N}(N(x-\theta), N(y-\theta)) .
\end{aligned}
$$

From (3.6), we deduce

$$
\mathrm{L}^{N}(x, x)=(1 / \sqrt{2})\left\{\psi_{N-1}\left(x_{N}\right) \psi_{N}^{\prime}\left(x_{N}\right)-\psi_{N}\left(x_{N}\right) \psi_{N-1}^{\prime}\left(x_{N}\right)\right\}
$$

Using the Schwartz inequality to (3.5), we see from (3.6) and (3.8) that

$$
\mathrm{L}^{N}(y, z)^{2} \leq \mathrm{L}^{N}(y, y) \mathrm{L}^{N}(z, z) .
$$

From here on, we assume

$$
-\frac{2}{3}<\alpha<-\frac{1}{2}
$$

We set

$$
\mathrm{B}^{N}=\left(-\sqrt{2}-N^{\alpha},-\sqrt{2}+N^{\alpha}\right) \cup\left(\sqrt{2}-N^{\alpha}, \sqrt{2}+N^{\alpha}\right) .
$$

The next lemma will be used in Sect. 4 .

Lemma 9 We set $\mathrm{U}^{N}=\mathbb{R} \backslash \mathrm{B}^{N}$. Then, the following holds.

(1) There exists a constant $c_{5}$ such that for all $N \in \mathbb{N}$

$$
\begin{aligned}
& \sup _{x, y \in \mathbb{R}}\left|L^{N}(x, y)\right| \leq c_{5} N^{\frac{1}{3}}, \\
& \sup _{x, y \in \mathrm{U}^{N}}\left|L^{N}(x, y)\right| \leq c_{5} .
\end{aligned}
$$

(2) Assume (3.12). Then, there exists a constant $c_{6}$ such that

$$
\left|L^{N}(x, y)\right| \leq \frac{c_{6}}{N|x-y|} \quad \text { for each } x, y \in \cup^{N}, N \in \mathbb{N} .
$$

Proof It is well known that

$$
\sqrt{2} \psi_{n}^{\prime}(x)=\sqrt{n} \psi_{n-1}(x)-\sqrt{n+1} \psi_{n+1}(x)
$$

From this and (3.10), we see that with a simple calculation

$$
\mathrm{L}^{N}(x, x)=\frac{1}{\sqrt{2}}\left\{\psi_{N-1} \psi_{N}^{\prime}-\psi_{N} \psi_{N-1}^{\prime}\right\}\left(x_{N}\right)
$$




$$
=\frac{N^{\frac{1}{2}}}{2}\left\{\psi_{N-1}^{2}+\psi_{N}^{2}-\sqrt{1-N^{-1}} \psi_{N-2} \psi_{N}-\sqrt{1+N^{-1}} \psi_{N-1} \psi_{N+1}\right\}\left(x_{N}\right) .
$$

Combining this with (3.2), we obtain

$$
\mathrm{L}^{N}(x, x) \leq \frac{N^{\frac{1}{2}}}{2} 5 c_{4}^{2} N^{-\frac{1}{6}}=\frac{5 c_{4}^{2}}{2} N^{\frac{1}{3}}
$$

From this and (3.11), we deduce (3.14). From Lemma 7 and (3.17), we see that

$$
\sup _{N \in \mathbb{N}} \sup _{y \in \mathrm{U}^{N}} \mathrm{~L}^{N}(y, y)<\infty .
$$

We deduce (3.15) from this and (3.11). Taking a constant $c_{5}$ in (3.14) and (3.15) in common completes the proof of (1).

Claim (3.16) follows from Lemma 7, (3.6), and (3.8).

\section{Proof of (2.21)-(2.24)}

As we see in Sect. 2, the point of the proof of Theorem 1.1 is to check conditions (2.21)-(2.24) in Lemma 6. The purpose of this section is to prove these equations. We recall a property of the reduced Palm measures of determinantal point processes.

Lemma 10 ([20]) Let $\mu$ be a determinantal point process with kernel $K$. Assume that $K(x, y)=\overline{K(y, x)}$ and $0 \leq \operatorname{Spec}(K) \leq 1$. Then, the reduced Palm measure $\mu_{x}$ is a determinantal point process with kernel $K_{x}$ given by

$$
K_{x}(y, z)=K(y, z)-\frac{K(y, x) K(x, z)}{K(x, x)}
$$

for $x$ such that $K(x, x)>0$.

Let $\mathrm{K}_{\theta}^{N}$ be the determinantal kernel of $\mu_{\theta}^{N}$ given by (3.7). Let $\mu_{\theta, x}^{N}$ be as in Lemma 6. Recall that $\mathrm{K}_{\theta}^{N}(y, z)=\mathrm{K}_{\theta}^{N}(z, y)$ by definition. Then, from this, (3.7), and (4.1), $\mu_{\theta, x}^{N}$ is a determinantal point process with kernel

$$
\mathrm{K}_{\theta, x}^{N}(y, z)=\mathrm{K}_{\theta}^{N}(y, z)-\frac{\mathrm{K}_{\theta}^{N}(x, y) \mathrm{K}_{\theta}^{N}(x, z)}{\mathrm{K}_{\theta}^{N}(x, x)} .
$$

From (3.4) and (4.2), we calculate correlation functions in (2.21)-(2.24) as follows.

$$
\begin{aligned}
& \rho_{\theta}^{N, 1}(y)=\mathrm{K}_{\theta}^{N}(y, y), \\
& \rho_{\theta, x}^{N, 1}(y)-\rho_{\theta}^{N, 1}(y)=-\frac{\mathrm{K}_{\theta}^{N}(x, y)^{2}}{\mathrm{~K}_{\theta}^{N}(x, x)},
\end{aligned}
$$




$$
\begin{aligned}
& \rho_{\theta}^{N, 2}(y, z)-\rho_{\theta}^{N, 1}(y) \rho_{\theta}^{N, 1}(z)=-\mathbf{K}_{\theta}^{N}(y, z)^{2}, \\
& \rho_{\theta, x}^{N, 2}(y, z)-\rho_{\theta, x}^{N, 1}(y) \rho_{\theta, x}^{N, 1}(z)-\left\{\rho_{\theta}^{N, 2}(y, z)-\rho_{\theta}^{N, 1}(y) \rho_{\theta}^{N, 1}(z)\right\} \\
& \quad=-\mathbf{K}_{\theta, x}^{N}(y, z)^{2}+\mathbf{K}_{\theta}^{N}(y, z)^{2} \\
& \quad=2 \frac{\mathbf{K}_{\theta}^{N}(y, z) \mathbf{K}_{\theta}^{N}(x, y) \mathbf{K}_{\theta}^{N}(x, z)}{\mathbf{K}_{\theta}^{N}(x, x)}-\frac{\mathbf{K}_{\theta}^{N}(x, y)^{2} \mathbf{K}_{\theta}^{N}(x, z)^{2}}{\mathbf{K}_{\theta}^{N}(x, x)^{2}} .
\end{aligned}
$$

Using these and (3.9), we rewrite (2.21)-(2.24) as follows.

Lemma 11 To simplify the notation, let

$$
x_{\theta}^{N}=\frac{x}{N}+\theta, \quad T_{r, \infty}^{N}(x)=\left\{y \in \mathbb{R} ; \frac{r}{N} \leq\left|x_{\theta}^{N}-y\right|<\infty\right\} .
$$

Then, (2.21)-(2.24) are equivalent to (4.8)-(4.11) below, respectively.

$$
\begin{aligned}
& \lim _{r \rightarrow \infty} \limsup _{N \rightarrow \infty}\left\|\int_{T_{r, \infty}^{N}(x)} \frac{L^{N}(y, y)}{x_{\theta}^{N}-y} d y-\theta\right\|_{R}=0, \\
& \lim _{r \rightarrow \infty} \limsup _{N \rightarrow \infty}\left\|\int_{T_{r, \infty}^{N}(x)} \frac{1}{x_{\theta}^{N}-y} \frac{L^{N}\left(x_{\theta}^{N}, y\right)^{2}}{L^{N}\left(x_{\theta}^{N}, x_{\theta}^{N}\right)} d y\right\|_{R}=0, \\
& \lim _{r \rightarrow \infty} \limsup _{N \rightarrow \infty}\left\|\int_{T_{r, \infty}^{N}(x)} \frac{1}{N} \frac{L^{N}(y, y)}{\left|x_{\theta}^{N}-y\right|^{2}} d y-\int_{T_{r, \infty}^{N}(x)^{2}} \frac{L^{N}(y, z)^{2}}{\left(x_{\theta}^{N}-y\right)\left(x_{\theta}^{N}-z\right)} \mathrm{d} y \mathrm{~d} z\right\|_{R}=0, \\
& \quad \lim _{r \rightarrow \infty} \limsup _{N \rightarrow \infty} \| \int_{T_{r, \infty}^{N}(x)} \frac{1}{N} \frac{1}{\left|x_{\theta}^{N}-y\right|^{2}} \frac{L^{N}\left(x_{\theta}^{N}, y\right)^{2}}{L^{N}\left(x_{\theta}^{N}, x_{\theta}^{N}\right)} \mathrm{d} y \\
& \quad+\int_{T_{r, \infty}^{N}(x)^{2}} \frac{1}{\left(x_{\theta}^{N}-y\right)\left(x_{\theta}^{N}-z\right)} \\
& \quad \times\left\{2 \frac{L^{N}(y, z) L^{N}\left(x_{\theta}^{N}, y\right) L^{N}\left(x_{\theta}^{N}, z\right)}{L^{N}\left(x_{\theta}^{N}, x_{\theta}^{N}\right)}-\frac{L^{N}\left(x_{\theta}^{N}, y\right) L^{N}\left(x_{\theta}^{N}, z\right)}{L^{N}\left(x_{\theta}^{N}, x_{\theta}^{N}\right)^{2}}\right\} \mathrm{d} y \mathrm{~d} z \|_{R}=0 .
\end{aligned}
$$

Proof Recall that $\mathrm{L}^{N}(x, y)=\mathrm{K}_{\theta}^{N}(N(x-\theta), N(y-\theta))$ by (3.9). Then, Lemma 11 follows easily from (4.3)-(4.6).

Let $\mathrm{B}^{N}$ and $\mathrm{U}^{N}$ be as in Lemma 9. Decompose $\mathrm{U}^{N}$ into $\mathrm{U}_{1}^{N}$ and $\mathrm{U}_{2}^{N}$ such that

$$
\mathrm{U}_{1}^{N}=\left[-\sqrt{2}+N^{\alpha}, \sqrt{2}-N^{\alpha}\right], \quad \bigcup_{2}^{N}=\mathbb{R} \backslash\left(-\sqrt{2}-N^{\alpha}, \sqrt{2}+N^{\alpha}\right) .
$$

Then, clearly $\mathrm{U}^{N}=\bigcup_{1}^{N} \cup \mathrm{U}_{2}^{N}$ and $\mathbb{R}=\bigcup_{1}^{N} \cup \mathrm{U}_{2}^{N} \cup \mathrm{B}^{N}$. We begin by the integral outside $\mathrm{U}_{1}^{N}$. 
Lemma 12 Let $0<q<3 / 2$. Then,

$$
\limsup _{N \rightarrow \infty}\left\|\int_{\mathbb{R} \backslash \cup_{1}^{N}} \frac{L^{N}(y, y)^{q}}{\left|x_{\theta}^{N}-y\right|} \mathrm{d} y\right\|_{R}=0 .
$$

Proof From (3.14), (4.7), and the definition of $\mathrm{B}^{N}$, we obtain that

$$
\begin{aligned}
& \limsup _{N \rightarrow \infty}\left\|\int_{\mathrm{B}^{N}} \frac{\mathrm{L}^{N}(y, y)^{q}}{\left|\mathrm{x}_{\theta}^{N}-y\right|} \mathrm{d} y\right\|_{R} \\
\leq & \limsup _{N \rightarrow \infty}\left\|\int_{\mathrm{B}^{N}} \frac{c_{5}^{q} N^{\frac{q}{3}}}{\left|\mathrm{x}_{\theta}^{N}-y\right|} \mathrm{d} y\right\|_{R} \\
\leq & \limsup _{N \rightarrow \infty} c_{5}^{q} N^{\frac{q}{3}}\left\{\log \left|\frac{x}{N}+\theta-\left(\sqrt{2}-N^{\alpha}\right)\right|-\log \left|\frac{x}{N}+\theta-\left(\sqrt{2}+N^{\alpha}\right)\right|\right\} \\
& +c_{5}^{q} N^{\frac{q}{3}}\left\{\log \left|\frac{x}{N}+\theta-\left(-\sqrt{2}-N^{\alpha}\right)\right|-\log \left|\frac{x}{N}+\theta-\left(-\sqrt{2}+N^{\alpha}\right)\right|\right\} \\
= & \mathcal{O}\left(N^{\frac{q}{3}+\alpha}\right)=0 \quad \text { as } N \rightarrow \infty .
\end{aligned}
$$

Here, we used $q<3 / 2$ and $\alpha<-1 / 2$ in the last line.

Note that $|y| \geq \sqrt{2}+N^{\alpha}$ for $y \in \bigcup_{2}^{N}$. Let $y=\sqrt{2} \cosh \tau$. Then, we see that

$$
\begin{aligned}
N \sinh ^{3} \tau & =N\left(\cosh ^{2} \tau-1\right)^{\frac{3}{2}} \\
& =N 2^{-\frac{3}{2}}\left(y^{2}-2\right)^{\frac{3}{2}} \geq N 2^{-\frac{3}{2}}\left(2 \sqrt{2} N^{\alpha}+N^{2 \alpha}\right)^{\frac{3}{2}}
\end{aligned}
$$

From this, $q>0$, and $\alpha>-2 / 3$, we apply (3.1) to obtain $c_{7}>0$ such that,

$$
\limsup _{N \rightarrow \infty}\left\|\int_{\mathrm{U}_{2}^{N}} \frac{\mathrm{L}^{N}(y, y)^{q}}{\left|\mathbf{x}_{\theta}^{N}-y\right|} \mathrm{d} y\right\|_{R} \leq \limsup _{N \rightarrow \infty}\left\|\int_{\mathrm{U}_{2}^{N}} \frac{c_{7}}{\left|\mathbf{x}_{\theta}^{N}-y\right| N^{q}\left(y^{2}-2\right)^{q}} \mathrm{~d} y\right\|_{R}=0
$$

which combined with (4.13) yields (4.12).

Lemma 13 (4.8) holds.

Proof Let $y=\sqrt{2} \cos \tau$. Then, $N \sin ^{3} \tau \geq N 2^{-\frac{3}{2}}\left(2 \sqrt{2} N^{\alpha}-N^{2 \alpha}\right)$ for $y \in \bigcup_{1}^{N}$. Then, applying Lemma 8 (1) we deduce that for each $r>0$

$$
\begin{aligned}
& \limsup _{N \rightarrow \infty}\left\|\int_{T_{r, \infty}^{N}(x) \cap \cup_{1}^{N}} \frac{\mathrm{L}^{N}(y, y)}{\mathbf{x}_{\theta}^{N}-y} \mathrm{~d} y-\theta\right\|_{R} \\
& =\limsup _{N \rightarrow \infty}\left\|\left\{\int_{-\sqrt{2}+N^{\alpha}}^{\mathbf{x}_{\theta}^{N}-\frac{r}{N}}+\int_{\mathbf{x}_{\theta}^{N}+\frac{r}{N}}^{\sqrt{2}-N^{\alpha}}\right\} \frac{1}{\mathbf{x}_{\theta}^{N}-y} \frac{1}{\pi} \sqrt{2-y^{2}} \mathrm{~d} y-\theta\right\|_{R} \\
& =\left|\mathrm{P} . \mathrm{V} \cdot \int_{-\sqrt{2}}^{\sqrt{2}} \frac{1}{\theta-y} \frac{1}{\pi} \sqrt{2-y^{2}} \mathrm{~d} y-\theta\right|=0 \text {. }
\end{aligned}
$$

Combining this with (4.12), we obtain (4.8). 
It is well known that $\mathrm{K}_{\theta}^{N}(x, x)$ are positive and continuous in $x$, and $\left\{\mathrm{K}_{\theta}^{N}(x, x)\right\}_{N \in \mathbb{N}}$ converges to $\mathrm{K}_{\theta}(x, x)=\sqrt{2-\theta^{2}} / \pi$ uniformly on each compact set. Then, we see

$$
\sup _{N \in \mathbb{N}} \sup _{x \in S_{R}} \frac{1}{\mathrm{~K}_{\theta}^{N}(x, x)}<\infty \text {. }
$$

From this, (3.9), and (4.7), we see that the following constant $c_{8}$ is finite.

$$
c_{8}:=\sup _{N \in \mathbb{N}} \sup _{x \in S_{R}} \frac{1}{L^{N}\left(\mathbf{x}_{\theta}^{N}, \mathbf{x}_{\theta}^{N}\right)}<\infty .
$$

Lemma 14 (4.15) and (4.16) below hold. In particular, (4.9) holds.

$$
\begin{aligned}
& \lim _{r \rightarrow \infty} \limsup _{N \rightarrow \infty}\left\|\int_{T_{r, \infty}^{N}(x)} \frac{L^{N}\left(x_{\theta}^{N}, y\right)^{2}}{\left|x_{\theta}^{N}-y\right| L^{N}\left(x_{\theta}^{N}, x_{\theta}^{N}\right)} \mathrm{d} y\right\|_{R}=0, \\
& \lim _{r \rightarrow \infty} \limsup _{N \rightarrow \infty}\left\|\int_{T_{r, \infty}^{N}(x)} \frac{L^{N}\left(x_{\theta}^{N}, y\right)}{\left|x_{\theta}^{N}-y\right| L^{N}\left(x_{\theta}^{N}, x_{\theta}^{N}\right)} \mathrm{d} y\right\|_{R}=0 .
\end{aligned}
$$

Proof From (3.11) and (4.12), we deduce that as $N \rightarrow \infty$

$$
\left\|\int_{\mathbb{R} \backslash \cup_{1}^{N}} \frac{\mathrm{L}^{N}\left(\mathbf{x}_{\theta}^{N}, y\right)^{2}}{\left|\mathbf{x}_{\theta}^{N}-y\right| \mathrm{L}^{N}\left(\mathbf{x}_{\theta}^{N}, \mathbf{x}_{\theta}^{N}\right)} \mathrm{d} y\right\|_{R} \leq\left\|\int_{\mathbb{R} \backslash \cup_{1}^{N}} \frac{\mathrm{L}^{N}(y, y)}{\left|\mathbf{x}_{\theta}^{N}-y\right|} d y\right\|_{R} \rightarrow 0 .
$$

From (3.16) and (4.14), for each $N \in \mathbb{N}$ and $r>0$

$$
\begin{aligned}
\left\|\int_{T_{r, \infty}^{N}(x) \cap \cup_{1}^{N}} \frac{\mathrm{L}^{N}\left(\mathbf{x}_{\theta}^{N}, y\right)^{2} d y}{\left|\mathbf{X}_{\theta}^{N}-y\right| \mathrm{L}^{N}\left(\mathbf{x}_{\theta}^{N}, \mathbf{x}_{\theta}^{N}\right)}\right\|_{R} & \leq\left\|\int_{T_{r, \infty}^{N}(x) \cap \cup_{1}^{N}} \frac{c_{6}^{2} c_{8} \mathrm{~d} y}{N^{2}\left|\mathbf{X}_{\theta}^{N}-y\right|^{3}}\right\|_{R} \\
& \leq \frac{c_{6}^{2} c_{8}}{r^{2}}
\end{aligned}
$$

Hence, (4.15) follows from (4.17) and (4.18). This completes the proof of (4.15).

We next prove (4.16). From (3.11), (4.12), and (4.14), we see for each $r>0$

$$
\limsup _{N \rightarrow \infty}\left\|\int_{T_{r, \infty}^{N}(x) \backslash \bigcup_{1}^{N}} \frac{\mathrm{L}^{N}\left(\mathbf{x}_{\theta}^{N}, y\right)}{\left|\mathbf{x}_{\theta}^{N}-y\right| \mathrm{L}^{N}\left(\mathbf{x}_{\theta}^{N}, \mathbf{x}_{\theta}^{N}\right)} d y\right\|_{R}=0 .
$$

From (3.16) and (4.14), we see that for each $N \in \mathbb{N}$ and $r>0$

$$
\begin{aligned}
\left\|\int_{T_{r, \infty}^{N}(x) \cap \cup_{1}^{N}} \frac{\mathrm{L}^{N}\left(\mathbf{x}_{\theta}^{N}, y\right) d y}{\left|\mathbf{x}_{\theta}^{N}-y\right| \mathrm{L}^{N}\left(\mathbf{x}_{\theta}^{N}, \mathbf{x}_{\theta}^{N}\right)}\right\|_{R} & \leq\left\|\int_{T_{r, \infty}^{N}(x) \cap \cup_{1}^{N}} \frac{c_{6} c_{8} \mathrm{~d} y}{N\left|\mathbf{x}_{\theta}^{N}-y\right|^{2}}\right\|_{R} \\
& \leq \frac{2 c_{6} c_{8}}{r}
\end{aligned}
$$

Combining (4.19) and (4.20), we obtain (4.16). 
Lemma 15 (4.21) and (4.22) hold. In particular, (4.10) holds.

$$
\begin{aligned}
& \lim _{r \rightarrow \infty} \limsup _{N \rightarrow \infty}\left\|\int_{T_{r, \infty}^{N}(x)} \frac{L^{N}(y, y)}{N\left|x_{\theta}^{N}-y\right|^{2}} \mathrm{~d} y\right\|_{R}=0, \\
& \lim _{r \rightarrow \infty} \limsup _{N \rightarrow \infty}\left\|\int_{T_{r, \infty}^{N}(x)^{2}} \frac{L^{N}(y, z)^{2}}{\left|x_{\theta}^{N}-y\right|\left|x_{\theta}^{N}-z\right|} \mathrm{d} y \mathrm{~d} z\right\|_{R}=0 .
\end{aligned}
$$

Proof Note that $\mathrm{L}^{N}(y, y) \leq c_{5}$ on $\mathrm{U}^{N}$ by (3.15). Then, by the definition of $T_{r, \infty}^{N}(x)$,

$$
\int_{T_{r, \infty}^{N}(x) \cap \bigcup^{N}} \frac{\mathrm{L}^{N}(y, y)}{N\left|\mathbf{x}_{\theta}^{N}-y\right|^{2}} \mathrm{~d} y \leq \frac{c_{5}}{N} \frac{2 N}{r}=\frac{2 c_{5}}{r} .
$$

By (3.14), we see $\mathrm{L}^{N}(y, y) \leq c_{5} N^{\frac{1}{3}}$ on $\mathbb{R}$. Recall that $\left|\mathrm{B}^{N}\right|=4 N^{\alpha}$ by construction. Furthermore, $c_{9}:=\limsup _{N \rightarrow \infty} \sup _{y \in \mathrm{B}^{N}}\left\|\left|\mathbf{x}_{\theta}^{N}-y\right|^{-2}\right\|_{R}<\infty$. Hence, for each $r>0$

$$
\limsup _{N \rightarrow \infty} \int_{T_{r, \infty}^{N}(x) \cap \mathrm{B}^{N}} \frac{\mathrm{L}^{N}(y, y)}{N\left|\mathbf{x}_{\theta}^{N}-y\right|^{2}} d y \leq \limsup _{N \rightarrow \infty} \frac{c_{5} N^{\frac{1}{3}} 4 N^{\alpha} c_{9}}{N}=0 .
$$

Here, we used $\alpha<-1 / 2$. We thus obtain (4.21) from (4.23) and (4.24).

We proceed with the proof of (4.22). We first consider the integral away from the diagonal line. By (3.16) and the Schwartz inequality, we see that

$$
\begin{aligned}
& \left\|\int_{\left(T_{r, \infty}^{N}(x) \cap \mathrm{U}^{N}\right)^{2} \cap\left\{|y-z| \geq \frac{1}{N}\right\}} \frac{\mathrm{L}^{N}(y, z)^{2}}{\left|\mathbf{X}_{\theta}^{N}-y\right|\left|\mathbf{X}_{\theta}^{N}-z\right|} \mathrm{d} y \mathrm{~d} z\right\|_{R} \\
& \leq\left\|\int_{\left(T_{r, \infty}^{N}(x) \cap U^{N}\right)^{2} \cap\left\{|y-z| \geq \frac{1}{N}\right\}} \frac{c_{6}^{2}}{N^{2}|y-z|^{2}\left|\mathbf{X}_{\theta}^{N}-y\right|\left|\mathbf{X}_{\theta}^{N}-z\right|} \mathrm{d} y \mathrm{~d} z\right\|_{R} \\
& \leq \|\left\{\int_{T_{r, \infty}^{N}(x)^{2} \cap\left\{|y-z| \geq \frac{1}{N}\right\}} \frac{c_{6}^{2}}{N^{2}|y-z|^{2}\left|\mathbf{X}_{\theta}^{N}-y\right|^{2}} \mathrm{~d} y \mathrm{~d} z\right\}^{\frac{1}{2}} \\
& \quad\left\{\int_{T_{r, \infty}^{N}(x)^{2} \cap\left\{|y-z| \geq \frac{1}{N}\right\}} \frac{c_{6}^{2}}{N^{2}|y-z|^{2}\left|\mathbf{X}_{\theta}^{N}-z\right|^{2}} \mathrm{~d} y \mathrm{~d} z\right\}^{\frac{1}{2}} \|_{R} \\
& \quad\left\|\int_{T_{r, \infty}^{N}(x)^{2} \cap\left\{|y-z| \geq \frac{1}{N}\right\}} \frac{c_{6}^{2}}{N^{2}|y-z|^{2}\left|\mathbf{X}_{\theta}^{N}-y\right|^{2}} \mathrm{~d} y \mathrm{~d} z\right\|_{R} \\
& \leq c_{6}^{2} \frac{2 N}{N^{2}}\left\{\frac{2 N}{r}\right\}=\frac{4 c_{6}^{2}}{r} .
\end{aligned}
$$

The last line follows from a straightforward calculation. Indeed, first integrating $z$ over $\left\{|y-z| \geq \frac{1}{N}\right\}$, and then integrating $y$ over $T_{r, \infty}^{N}(x)$, we obtain the inequality in the 
last line. We therefore see that

$$
\lim _{r \rightarrow \infty} \lim _{N \rightarrow \infty}\left\|\int_{\left(T_{r, \infty}^{N}(x) \cap U^{N}\right)^{2} \cap\left\{|y-z| \geq \frac{1}{N}\right\}} \frac{\mathrm{L}^{N}(y, z)^{2}}{\left|\mathbf{X}_{\theta}^{N}-y\right|\left|\mathbf{X}_{\theta}^{N}-z\right|} \mathrm{d} y \mathrm{~d} z\right\|_{R}=0 .
$$

We next consider the integral near the diagonal. From (3.15), we see that

$$
\begin{aligned}
& \left\|\int_{\left(T_{r, \infty}^{N}(x) \cap \mathrm{U}^{N}\right)^{2} \cap\left\{|y-z| \leq \frac{1}{N}\right\}} \frac{\mathrm{L}^{N}(y, z)^{2}}{\left|\mathbf{X}_{\theta}^{N}-y\right|\left|\mathbf{X}_{\theta}^{N}-z\right|} \mathrm{d} y \mathrm{~d} z\right\|_{R} \\
& \leq\left\|\int_{\left(T_{r, \infty}^{N}(x) \cap U^{N}\right)^{2} \cap\left\{|y-z| \leq \frac{1}{N}\right\}} \frac{c_{5}^{2}}{\left|\mathbf{X}_{\theta}^{N}-y\right|\left|\mathbf{X}_{\theta}^{N}-z\right|} \mathrm{d} y \mathrm{~d} z\right\|_{R} \\
& \leq\left\|\int_{T_{r, \infty}^{N}(x)^{2} \cap\left\{|y-z| \leq \frac{1}{N}\right\}} \frac{c_{5}^{2}}{2}\left\{\frac{1}{\left|\mathbf{X}_{\theta}^{N}-y\right|^{2}}+\frac{1}{\left|\mathbf{X}_{\theta}^{N}-z\right|^{2}}\right\} \mathrm{d} y \mathrm{~d} z\right\|_{R} \\
& =\frac{2 c_{5}^{2}}{N}\left\|\int_{T_{r, \infty}^{N}(x)} \frac{1}{\left|\mathbf{X}_{\theta}^{N}-y\right|^{2}} \mathrm{~d} y\right\|_{R}=\frac{2 c_{5}^{2}}{N} \frac{2 N}{r}=\frac{4 c_{5}^{2}}{r} .
\end{aligned}
$$

From (4.25) and (4.26), we have

$$
\lim _{r \rightarrow \infty} \lim _{N \rightarrow \infty}\left\|\int_{\left(T_{r, \infty}^{N}(x) \cap U^{N}\right)^{2}} \frac{\mathrm{L}^{N}(y, z)^{2}}{\left|\mathbf{X}_{\theta}^{N}-y\right|\left|\mathbf{X}_{\theta}^{N}-z\right|} \mathrm{d} y \mathrm{~d} z\right\|_{R}=0 .
$$

We next consider the integral on $\mathrm{B}^{N} \times \mathrm{B}^{N}$. Let

$$
c_{10}=\limsup _{N \rightarrow \infty} \sup _{x \in S_{R}, y \in \mathrm{B}^{N}}\left|\mathbf{x}_{\theta}^{N}-y\right|^{-1} .
$$

Then, we deduce from (3.14) and the definition of $\mathrm{B}^{N}$ given by (3.13) that

$$
\begin{aligned}
\limsup _{N \rightarrow \infty} & \left\|\int_{\left(T_{r, \infty}^{N}(x) \cap \mathrm{B}^{N}\right)^{2}} \frac{\mathrm{L}^{N}(y, z)^{2}}{\left|\mathbf{x}_{\theta}^{N}-y\right|\left|\mathbf{x}_{\theta}^{N}-z\right|} \mathrm{d} y \mathrm{~d} z\right\|_{R} \\
\leq & \lim _{N \rightarrow \infty} c_{5}^{2} c_{10}^{2} N^{\frac{2}{3}}\left(4 N^{\alpha}\right)^{2}=0 .
\end{aligned}
$$

Here, we used $\left|\mathrm{B}^{N}\right|=4 N^{\alpha}$ for the inequality and $\alpha<-1 / 2$ for the last equality.

We finally consider the case $\mathrm{U}^{N} \times \mathrm{B}^{N}$. Then, a similar argument yields

$$
\begin{aligned}
& \left\|\int_{\left(T_{r, \infty}^{N}(x) \cap \mathrm{U}^{N}\right) \times\left(T_{r, \infty}^{N}(x) \cap \mathrm{B}^{N}\right)} \frac{\mathrm{L}^{N}(y, z)^{2}}{\left|\mathbf{X}_{\theta}^{N}-y\right|\left|\mathbf{x}_{\theta}^{N}-z\right|} \mathrm{d} y \mathrm{~d} z\right\|_{R} \\
& \quad \leq\left\|\int_{\left(T_{r, \infty}^{N}(x) \cap \mathrm{U}^{N}\right) \times\left(T_{r, \infty}^{N}(x) \cap \mathrm{B}^{N}\right)} \frac{\mathrm{L}^{N}(y, y) \mathrm{L}^{N}(z, z)}{\left|\mathbf{X}_{\theta}^{N}-y\right|\left|\mathbf{X}_{\theta}^{N}-z\right|} \mathrm{d} y \mathrm{~d} z\right\|_{R} \\
& \quad=\left\|\int_{T_{r, \infty}^{N}(x) \cap \mathrm{U}^{N}} \frac{\mathrm{L}^{N}(y, y)}{\left|\mathbf{X}_{\theta}^{N}-y\right|} \mathrm{d} y \int_{T_{r, \infty}^{N}(x) \cap \mathrm{B}^{N}} \frac{\mathrm{L}^{N}(z, z)}{\left|\mathbf{X}_{\theta}^{N}-z\right|} \mathrm{d} z\right\|_{R}
\end{aligned}
$$




$$
=\mathcal{O}(\log N) \mathcal{O}\left(N^{\frac{1}{3}+\alpha}\right) \rightarrow 0 \quad \text { as } N \rightarrow \infty .
$$

Collecting (4.27), (4.28), and (4.29), we conclude (4.22).

\section{Lemma 16 (4.11) holds.}

Proof We shall estimate the three terms in (4.11) beginning with the first. From (3.11) and (4.21), we have

$$
\begin{aligned}
& \lim _{r \rightarrow \infty} \limsup _{N \rightarrow \infty}\left\|\int_{T_{r, \infty}^{N}(x)} \frac{\mathrm{L}^{N}\left(\mathbf{x}_{\theta}^{N}, y\right)^{2} \mathrm{~d} y}{N\left|\mathbf{x}_{\theta}^{N}-y\right|^{2} \mathrm{~L}^{N}\left(\mathbf{x}_{\theta}^{N}, \mathbf{x}_{\theta}^{N}\right)}\right\|_{R} \\
& \leq \lim _{r \rightarrow \infty} \limsup _{N \rightarrow \infty}\left\|\int_{T_{r, \infty}^{N}(x)} \frac{\mathrm{L}^{N}(y, y) \mathrm{d} y}{N\left|\mathbf{x}_{\theta}^{N}-y\right|^{2}}\right\|_{R}=0 .
\end{aligned}
$$

Next, using the Schwartz inequality, we have for the second term

$$
\begin{aligned}
& \left\|\int_{T_{r, \infty}^{N}(x)^{2}} \frac{\mathrm{L}^{N}(y, z) \mathrm{L}^{N}\left(\mathbf{x}_{\theta}^{N}, y\right) \mathrm{L}^{N}\left(\mathbf{x}_{\theta}^{N}, z\right) \mathrm{d} y \mathrm{~d} z}{\left|\mathbf{x}_{\theta}^{N}-y\right|\left|\mathbf{x}_{\theta}^{N}-z\right| \mathrm{L}^{N}\left(\mathbf{x}_{\theta}^{N}, \mathbf{x}_{\theta}^{N}\right)}\right\|_{R} \\
& \quad \leq\left\|\int_{T_{r, \infty}^{N}(x)^{2}} \frac{\mathrm{L}^{N}(y, z)^{2} \mathrm{~d} y \mathrm{~d} z}{\left|\mathbf{x}_{\theta}^{N}-y\right|\left|\mathbf{x}_{\theta}^{N}-z\right|}\right\|_{R}^{\frac{1}{2}}\left\|\int_{T_{r, \infty}^{N}(x)^{2}} \frac{\mathrm{L}^{N}\left(\mathbf{x}_{\theta}^{N}, y\right)^{2} \mathrm{~L}^{N}\left(\mathbf{x}_{\theta}^{N}, z\right)^{2} \mathrm{~d} y \mathrm{~d} z}{\left|\mathbf{x}_{\theta}^{N}-y\right|\left|\mathbf{x}_{\theta}^{N}-z\right| \mathrm{L}^{N}\left(\mathbf{x}_{\theta}^{N}, \mathbf{x}_{\theta}^{N}\right)^{2}}\right\|_{R}^{\frac{1}{2}} \\
& \quad=\left\|\int_{T_{r, \infty}^{N}(x)^{2}} \frac{\mathrm{L}^{N}(y, z)^{2} \mathrm{~d} y \mathrm{~d} z}{\left|\mathbf{x}_{\theta}^{N}-y\right|\left|\mathbf{x}_{\theta}^{N}-z\right|}\right\|_{R}^{\frac{1}{2}}\left\|\int_{T_{r, \infty}^{N}(x)} \frac{\mathrm{L}^{N}\left(\mathbf{x}_{\theta}^{N}, y\right)^{2}}{\left|\mathbf{x}_{\theta}^{N}-y\right| \mathrm{L}^{N}\left(\mathbf{x}_{\theta}^{N}, \mathbf{x}_{\theta}^{N}\right)} \mathrm{d} y\right\|_{R} \cdot
\end{aligned}
$$

Applying (4.22) and (4.15) to the last line, we obtain

$$
\lim _{r \rightarrow \infty} \limsup _{N \rightarrow \infty}\left\|\int_{T_{r, \infty}^{N}(x)^{2}} \frac{\mathrm{L}^{N}(y, z) \mathrm{L}^{N}\left(\mathbf{x}_{\theta}^{N}, y\right) \mathrm{L}^{N}\left(\mathbf{x}_{\theta}^{N}, z\right) \mathrm{d} y \mathrm{~d} z}{\left|\mathbf{x}_{\theta}^{N}-y \| \mathbf{x}_{\theta}^{N}-z\right| \mathrm{L}^{N}\left(\mathbf{x}_{\theta}^{N}, \mathbf{x}_{\theta}^{N}\right)}\right\|_{R}=0 .
$$

We finally estimate the third term. From (4.16), as $N \rightarrow \infty$, we have

$$
\begin{aligned}
& \left\|\int_{T_{r, \infty}^{N}(x)^{2}} \frac{\mathrm{L}^{N}\left(\mathbf{x}_{\theta}^{N}, y\right) \mathrm{L}^{N}\left(\mathbf{x}_{\theta}^{N}, z\right) \mathrm{d} y \mathrm{~d} z}{\left|\mathbf{x}_{\theta}^{N}-y\right|\left|\mathbf{x}_{\theta}^{N}-z\right| \mathrm{L}^{N}\left(\mathbf{x}_{\theta}^{N}, \mathbf{x}_{\theta}^{N}\right)^{2}}\right\|_{R} \\
& \quad=\left\|\left\{\int_{T_{r, \infty}^{N}(x)} \frac{\mathrm{L}^{N}\left(\mathbf{x}_{\theta}^{N}, y\right) \mathrm{d} y}{\left|\mathbf{x}_{\theta}^{N}-y\right| \mathrm{L}^{N}\left(\mathbf{x}_{\theta}^{N}, \mathbf{x}_{\theta}^{N}\right)}\right\}^{2}\right\|_{R} \\
& =\left\|\int_{T_{r, \infty}^{N}(x)} \frac{\mathrm{L}^{N}\left(\mathbf{x}_{\theta}^{N}, y\right) \mathrm{d} y}{\left|\mathbf{x}_{\theta}^{N}-y\right| \mathrm{L}^{N}\left(\mathbf{x}_{\theta}^{N}, \mathbf{x}_{\theta}^{N}\right)}\right\|_{R}^{2} \rightarrow 0 \quad \text { by (4.16). }
\end{aligned}
$$

From (4.30), (4.31), and (4.32), we obtain (4.11). This completes the proof. 


\section{Proof of Theorem 1.1}

From Lemmas 13-16, we deduce that all the assumptions (2.21)-(2.24) in Lemma 6 are satisfied. Hence, (2.10) is proved by Lemma 6. Then, Theorem 1.1 follows from Lemmas 4, 5, and 6.

\section{Proof of Theorem 1.2}

In this section, we prove Theorem 1.2 using Theorem 1.1. It is sufficient for the proof of Theorem 1.2 to prove (1.15) in $C\left([0, T] ; \mathbb{R}^{m}\right)$ for each $T \in \mathbb{N}$. Hence, we fix $T \in \mathbb{N}$. Let $\mathbf{X}^{N}=\left(X^{N, i}\right)_{i=1}^{N}$ be as in (1.13). Let $Y^{\theta, N, i}=\left\{Y_{t}^{\theta, N, i}\right\}$ such that

$$
Y_{t}^{\theta, N, i}=X_{t}^{N, i}+\theta t
$$

Then, from (1.13) we see that $\mathbf{Y}^{\theta, N}=\left(Y^{\theta, N, i}\right)_{i=1}^{N}$ is a solution of

$$
\mathrm{d} Y_{t}^{\theta, N, i}=\mathrm{d} B_{t}^{i}+\sum_{j \neq i}^{N} \frac{1}{Y_{t}^{\theta, N, i}-Y_{t}^{\theta, N, j}} \mathrm{~d} t-\frac{1}{N} Y_{t}^{\theta, N, i} \mathrm{~d} t+\frac{\theta}{N} \mathrm{~d} t
$$

with the same initial condition as $\mathbf{X}^{N}$. Let $P^{\theta, N}$ and $Q^{\theta, N}$ be the distributions of $\mathbf{X}^{N}$ and $\mathbf{Y}^{\theta, N}$ on $C\left([0, T] ; \mathbb{R}^{N}\right)$, respectively. Then, applying the Girsanov theorem $[3$, pp. 190-195] to (6.2), we see that

$$
\begin{aligned}
\frac{\mathrm{d} Q^{\theta, N}}{\mathrm{~d} P^{\theta, N}}(\mathbf{W})= & \exp \left\{\int_{0}^{T} \sum_{i=1}^{N} \frac{\theta}{N} \mathrm{~d} B_{t}^{i}-\frac{1}{2} \int_{0}^{T} \sum_{i=1}^{N}\left|\frac{\theta}{N}\right|^{2} \mathrm{~d} t\right\} \\
& =\exp \left\{\frac{\theta}{N} \sum_{i=1}^{N} B_{T}^{i}-\frac{\theta^{2} T}{2 N}\right\}
\end{aligned}
$$

where we write $\mathbf{W}=\left(W^{i}\right) \in C\left([0, T] ; \mathbb{R}^{N}\right)$ and $\left\{B^{i}\right\}_{i=1}^{N}$ under $P^{\theta, N}$ are independent copies of Brownian motions starting at the origin.

Lemma 17 For each $\epsilon>0$,

$$
\lim _{N \rightarrow \infty} Q^{\theta, N}\left(\left|\frac{\mathrm{d} P^{\theta, N}}{\mathrm{~d} Q^{\theta, N}}(\boldsymbol{W})-1\right| \geq \epsilon\right)=0 .
$$

Proof It is sufficient for (6.4) to prove, for each $\epsilon>0$,

$$
\lim _{N \rightarrow \infty} P^{\theta, N}\left(\left|\frac{\mathrm{d} Q^{\theta, N}}{d P^{\theta, N}}(\mathbf{W})-1\right| \geq \epsilon\right)=0 .
$$

This follows from (6.3) immediately. 
Proof of Theorem 1.2 We write $\mathbf{W}^{m}=\left(W^{1}, \ldots, W^{m}\right) \in C\left([0, T] ; \mathbb{R}^{m}\right)$ for $\mathbf{W}=$ $\left(W^{i}\right)_{i=1}^{N}$, where $m \leq N \leq \infty$. Let $Q^{\theta}$ be the distribution of the solution $\mathbf{Y}^{\theta}$ with initial distribution $\mu_{\theta} \circ \mathfrak{l}^{-1}$. From Theorem 1.1 and (6.1), we deduce that for each $m \in \mathbb{N}$

$$
\lim _{N \rightarrow \infty} Q^{\theta, N}\left(\mathbf{W}^{m} \in \cdot\right)=Q^{\theta}\left(\mathbf{W}^{m} \in \cdot\right)
$$

weakly in $C\left([0, T] ; \mathbb{R}^{m}\right)$. Then, from this, for each $F \in C_{b}\left(C\left([0, T] ; \mathbb{R}^{m}\right)\right)$,

$$
\lim _{N \rightarrow \infty} \int_{C\left([0, T] ; \mathbb{R}^{N}\right)} F\left(\mathbf{W}^{m}\right) \mathrm{d} Q^{\theta, N}=\int_{C\left([0, T] ; \mathbb{R}^{\mathbb{N}}\right)} F\left(\mathbf{W}^{m}\right) \mathrm{d} Q^{\theta} .
$$

We obtain from (6.4) and (6.5) that

$$
\begin{aligned}
\lim _{N \rightarrow \infty} \int_{C\left([0, T] ; \mathbb{R}^{N}\right)} F\left(\mathbf{W}^{m}\right) \mathrm{d} P^{N, \theta} & =\lim _{N \rightarrow \infty} \int_{C\left([0, T] ; \mathbb{R}^{N}\right)} F\left(\mathbf{W}^{m}\right) \frac{\mathrm{d} P^{\theta, N}}{\mathrm{~d} Q^{\theta, N}}(\mathbf{W}) \mathrm{d} Q^{\theta, N} \\
& =\lim _{N \rightarrow \infty} \int_{C\left([0, T] ; \mathbb{R}^{N}\right)} F\left(\mathbf{W}^{m}\right) \mathrm{d} Q^{\theta, N} \\
& =\int_{C\left([0, T] ; \mathbb{R}^{\mathbb{N}}\right)} F\left(\mathbf{W}^{m}\right) \mathrm{d} Q^{\theta} .
\end{aligned}
$$

This implies (1.15). We have thus completed the proof of Theorem 1.2.

Acknowledgements H.O. thanks Professor H. Spohn for a useful comment at RIMS in Kyoto University in 2002 .

Open Access This article is distributed under the terms of the Creative Commons Attribution 4.0 International License (http://creativecommons.org/licenses/by/4.0/), which permits unrestricted use, distribution, and reproduction in any medium, provided you give appropriate credit to the original author(s) and the source, provide a link to the Creative Commons license, and indicate if changes were made.

\section{References}

1. Anderson, G.W., Guionnet, A., Zeitouni, O.: An Introduction to Random Matrices. Cambridge University Press, Cambridge (2010)

2. Bourgade, P., Erdös, L., Yau, H.-T.: Universality of general $\beta$-ensembles. Duke Math. J. 163, 11271190 (2014)

3. Ikeda, N., Watanabe, S.: Stochastic differential equations and diffusion processes, 2nd edn. NorthHolland, Amsterdam (1989)

4. Inukai, K.: Collision or non-collision problem for interacting Brownian particles. Proc. Jpn. Acad. Ser. A 82, 66-70 (2006)

5. Katori, M., Tanemura, H.: Non-equilibrium dynamics of Dyson's model with an infinite number of particles. Commun. Math. Phys. 136, 1177-1204 (2010)

6. Katori, M., Tanemura, H.: Markov property of determinantal processes with extended sine. Airy Bessel Kernels Markov Process. Relat. Fields 17, 541-580 (2011)

7. Kawamoto, Y.: Density preservation of unlabeled diffusion in systems with infinitely many particles. In: Stochastic Analysis on Large Scale Interacting Systems, vol. B59, pp. 337-350. RIMS Kyoto (2016) 
8. Kawamoto, Y., Osada, H.: Finite-particle approximations for interacting Brownian particles with logarithmic potentials. to appear in J. Math. Soc. Jpn. arXiv:1607.06922v2 [math.PR]

9. Landon, B., Sosoe, P., Yau, H.-T.: Fixed energy universality of Dyson Brownian motion. arXiv:1609.09011

10. Mehta, M.L.: Random Matrices, 3rd edn. Elsevier, Amsterdam (2004)

11. Osada, H.: Non-collision and collision properties of Dyson's model in infinite dimensions and other stochastic dynamics whose equilibrium states are determinantal random point fields. In: Funaki T., Osada H. (eds.) Stochastic Analysis on Large Scale Interacting Systems. Advanced Studies in Pure Mathematics, vol. 39, pp. 325-343 (2004)

12. Osada, H.: Infinite-dimensional stochastic differential equations related to random matrices. Probab. Theory Relat. Fields 153, 471-509 (2012)

13. Osada, H.: Interacting Brownian motions in infinite dimensions with logarithmic interaction potentials. Ann. Probab. 41, 1-49 (2013)

14. Osada, H.: Interacting Brownian motions in infinite dimensions with logarithmic interaction potentials II: airy random point field. Stoch. Process. Appl. 123, 813-838 (2013)

15. Osada, H., Osada, S.: Discrete approximations of determinantal point processes on continuous spaces: tree representations and tail triviality. J. Stat. Phys. 170(2), 421-435 (2018)

16. Osada, H., Tanemura, H.: Strong Markov property of determinantal processes with extended kernels. Stoch. Process. Appl. 126(1), 186-208 (2016). https://doi.org/10.1016/j.spa.2015.08.003

17. Osada, H., Tanemura, H.: Infinite-dimensional stochastic differential equations and tail $\sigma$-fields. arXiv:1412.8674v5 [math.PR]

18. Osada, H., Tanemura, H.: Infinite-dimensional stochastic differential equations related to Airy random point fields. arXiv: 1408.0632

19. Plancherel, M., Rotach, W.: Sur les valeurs asymptotiques des polynomes dH́ermite $H_{n}(x)=$ $(-1)^{n} e^{x^{2} / 2} \frac{d^{n}}{d x^{n}}\left(e^{-x^{2} / 2}\right)$. Comment. Math. Helv. 1, 227-254 (1929)

20. Shirai, T., Takahashi, Y.: Random point fields associated with certain Fredholm determinants I: fermion, Poisson and boson point process. J. Funct. Anal. 205, 414-463 (2003)

21. Soshnikov, A.: Determinantal random point fields. Russ. Math. Surv. 55, 923-975 (2000)

22. Spohn, H.: Interacting Brownian particles: a study of Dyson's model. In: Papanicolaou, G. (ed.) Hydrodynamic Behavior and Interacting Particle Systems, IMA Volumes in Mathematics and its Applications, vol. 9, pp. 151-179. Springer, Berlin (1987)

23. Tsai, Li-Cheng: Infinite dimensional stochastic differential equations for Dyson's model. Probab. Theory Relat. Fields. 166(3-4), 801-850 (2016) 